\title{
Checklist of the coral fish fauna of Xisha Islands, China
}

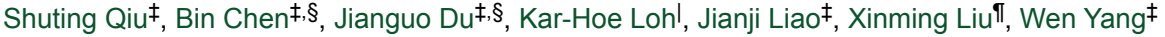 \\ ¥ Third Institute of Oceanography, Ministry of Natural Resources, Xiamen, China \\ $\S$ Fujian Provincial Key Laboratory ofMarine Ecological Conservation and Restoration, Xiamen, China \\ | Institute of Ocean and Earth Sciences, University of Malaya, Kuala Lumpur, Malaysia \\ II Guangxi University of Chinese Medicine, Guangxi, China
}

Corresponding author: Jianguo Du (dujianguo@tio.org.cn)

Academic editor: Yahui Zhao

Received: 04 Feb 2021 | Accepted: 01 Mar 2021 | Published: 08 Mar 2021

Citation: Qiu S, Chen B, Du J, Loh K-H, Liao J, Liu X, Yang W (2021) Checklist of the coral fish fauna of Xisha

Islands, China. Biodiversity Data Journal 9: e63945. https://doi.org/10.3897/BDJ.9.e63945

\section{Abstract}

\section{Background}

The Xisha Islands are composed of the Yongle Islands and the Xuande Islands in Hainan Province, China. It has one of the highest species diversity in the world and is also a typical oceanic distribution area of coral reefs globally. The ichthyofauna of the Xisha Islands were recorded by underwater visual census in May 2019 and July 2020. The survey data were combined with previous records of species into the checklist of the Xisha Islands presented herein. A total of 691 species, belonging to 24 orders and 97 families, was recorded. The major families were Labridae, Pomacentridae, Serranidae, Chaetodontidae, Hexanchidae, Lutjanidae, Scaridae, Gobiidae, Scorpaenidae and Carangidae. In this study, the Coral Fish iversity Index (CFDI) of six families (Chaetodontidae, Pomacanthidae, Pomacentridae, Labridae, Scaridae and Acanthuridae) was 229, indicating 756 coral fishes. In terms of the IUCN Red List, one species is Critically Endangered (Glyphis gangeticus), six species are Endangered (Stegostoma fasciatum, Aetomylaeus maculatus, Aetomylaeus vespertilio, Epinephelus akaara, Cheilinusundulatus sp. and Xiphias gladius), 16 species are Vulnerable, and 13 species are Near Threatened in the Xisha Archipelago, so conservation should be strengthened in this area in the future. 


\section{New information}

One species is a new record for China (Dischistodus pseudochrysopoecilus) and 23 species are newly found in the Xisha Islands.

\section{Keywords}

Ichthyofauna, Coral Fish Diversity Index (CFDI), Xisha Islands, newly-recorded species

\section{Introduction}

The Xisha Archipelago is in the midwest of the South China Sea and it is at the northern margin of the Coral Triangle, which has the highest biodiversity in the world (Allen 2008). The Xisha Islands comprise the Yongle Islands and the Xuande Islands and begins in the north from the Beijiao Atoll and ends at the Songtao Bank in the south; Xidu Bank is in the east and Zhongjian Island in the west. Altogether, there are 29 islands, sandbars and four atolls.

The coral reefs of the Xisha Islands are the oldest and most primitive in China. It is also the birthplace of the coral reef ecosystem in the coastal areas of China (Huang et al. 2020). The Xisha Coral Reef Ecological Monitoring Zone was established in 2005 by the State Oceanic Administration of China to monitor and assess the health of coral reefs. The coral reef coverage declined from $53.8 \%$ in 2007 to $5.44 \%$ in 2016 , due to anthropogenic activities, outbreak of crown-of-thorns starfish, coral diseases, typhoons and global warming (Li et al. 2017).

Several studies have focused on the fishes in the Xisha Islands. For example, 261 species belonging to 48 families of coral reef fishes were found in Beijiao Atoll, Yongxing Island, Huaguang Atoll and five other islands by gillnet and angling (Sun et al. 2004). A total of 146 species belonging to 31 families of fish was reported from the main reefs in Xisha Islands by bottom gillnet (Wang et al. 2011). There were 119 species belonging to 73 genera and 30 families of coral reef fish species reported in the Xisha Islands by underwater visual censuses and 643 species were recorded in combination with other previous studies (Gao et al. 2014). However, several new studies have been published. For example, in Zhaoshu Island, a total of 58 coral reef fish species was recorded, belonging to one class, three orders, 18 families and 37 genera by underwater visual censuses (Yang et al. 2018).The diversity of reef fishes declined from $3.10 / \mathrm{m}^{2}$ in 2005 to $1.23 / \mathrm{m}^{2}$ in 2013 , due to reef degradation and overfishing ( $\mathrm{Li}$ et al. 2017). The fish list of the Xisha Islands remains incomplete and taxonomic revisions are urgently needed to provide scientific support for follow-up research and protection of reef fishes in the Xisha Islands, especially against the background of intense human activity and rapid global change. 


\section{Materials and methods}

In May 2019 and July 2020, a total of 27 sites in the Xisha Islands (Fig. 1) was investigated by underwater visual censuses and more than 50 dives were performed, using a Canon 5D4, together with Seacam 150D and Sea\&Sea YS-D2 flashlights. The photos were taken ranging from 5 to $30 \mathrm{~m}$. The species were identified according to the following resources: Reef fishes of the East Indies (Allen and Erdmann 2012), Coral reef atlas of Xisha Islands (Huang 2018), Reef fish identification of Nansha Islands (Fang and Lv 2019), Coral reef fishes of the South China Sea (Fu 2014) and FishBase (Froese and Pauly 2020, Fig. 1)

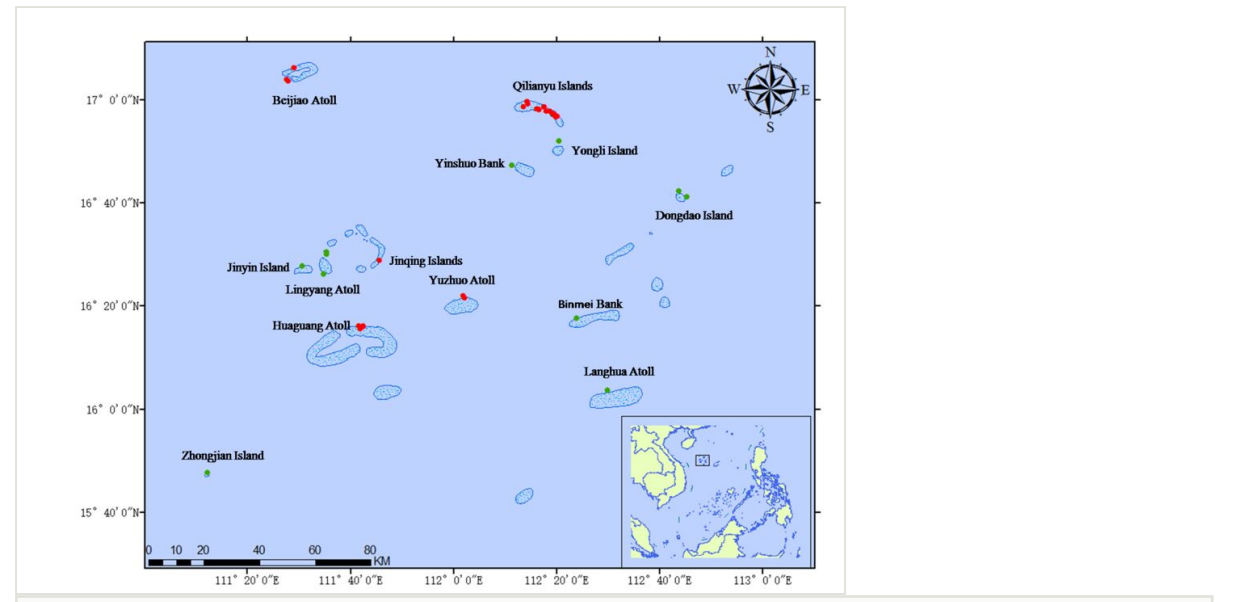

Figure 1. doi

The map showing the survey locations.

The Coral Fish Diversity Index (CFDI) is an evaluative index proposed by Allen (1988) to measure the diversity of coral reef fishes using the following formula: Total fish fauna = 3.39 (CFDI) - 20.595 used by for areas under $2,000 \mathrm{~km}$. The CFDI is based on the total number of species in each of the six indicator families (Acanthuridae, Chaetodontidae, Labridae, Pomacanthidae, Pomacentridae and Scaridae). All selected families are crucial parts of reef communities, widely distributed and firmly related to coral reef ecosystems.

This checklist has been arranged in the evolutionary order of class, order, family and species and families are arranged in the evolutionary order of genera and then species name. The newly-recorded species in the Xisha Archipelago are marked with $\mathrm{r} 11$ only in the Table. For habitats types, these abbreviations have been used: reef associated (RFA); brackish (BRA); demersal (DEM); amphidromous (AMP); pelagic (PEL); bathydemersal $(B A D)$; benthopelagic (BEP); bathypelagic (BAP); pelagic-neritic (PE); oceanodromous (OD).

The IUCN status is indicated as: Critically Endangered (CE); Endangered (EN); Vulnerable (VU); Near Threatened (NT); Lower Risk (LR); Least Concern (LC). Threat to humans: Other; Traumatogenic; Poisonous to eat; Venomous; Reports of ciguatera poisoning. 
There were 197 species belonging to 28 families found in this survey, of which, one species represented a new record in China (Dischistodus pseudochrysopoecilus) and 23 species represent new records in the Xisha Islands (Table 1), combined with eight previous studies by Gao et al. (2014) and Sun et al. (2004)Li et al. 2017, Li et al. 2007, Wang 1981, Wang et al. 2011, Yang et al. 2018, Zeng 2004. In total, 690 species were recorded from the Xisha Islands, belonging to 24 orders and 97 families. The order Perciformes was the most dominant order, with 496 species belonging to it. The most dominant family was the family Labridae, which had 75 species; followed by Pomacentridae with 63 species; the third, fourth and and fifth largest families were Serranidae, Chaetodontidae and Hexanchidae, which had 42 species, 39 species and 29 species, respectively. Other major families were Lutjanidae and Scaridae, which both had 26 species, Gobiidae had 22 species; Scorpaenidae and Carangidae had 16 species (Table 1).

\section{Table 1.}

Checklist of the coral fish fauna of the Xisha Islands, China.

\begin{tabular}{|c|c|c|c|c|c|}
\hline $\begin{array}{l}\text { Subclass, Order, Family, Genus, and } \\
\text { species }\end{array}$ & $\begin{array}{l}\text { Habitat } \\
\text { types }\end{array}$ & Threat to humans & $\begin{array}{l}\text { IUCN } \\
\text { status }\end{array}$ & $\begin{array}{l}\text { Trophic } \\
\text { level }\end{array}$ & $\begin{array}{l}\text { Other } \\
\text { remarks }\end{array}$ \\
\hline \multicolumn{6}{|l|}{ Class Chondrichthyes } \\
\hline \multicolumn{6}{|l|}{ Order Chimaeriformes } \\
\hline \multicolumn{6}{|l|}{ Family Chimaeridae } \\
\hline \multicolumn{6}{|l|}{ Genus Chimaera } \\
\hline $\begin{array}{l}\text { Chimaera phantasma Jordan \& Snyder, } \\
1900\end{array}$ & BAD & & & 3.5 & r1 \\
\hline \multicolumn{6}{|l|}{ Order Orectolobiformes } \\
\hline \multicolumn{6}{|l|}{ Family Orectolobidae } \\
\hline \multicolumn{6}{|l|}{ Elasmobranchii } \\
\hline \multicolumn{6}{|l|}{ Order Orectolobiformes } \\
\hline \multicolumn{6}{|l|}{ Genus Stegostoma } \\
\hline Stegostoma fasciatum (Hermann, 1783) & RFA & Traumatogenic & EN & 3.1 & r1 \\
\hline \multicolumn{6}{|l|}{ Family Ginglymostomatidae } \\
\hline \multicolumn{6}{|l|}{ Genus Nebrius } \\
\hline Nebrius ferrugineus (Lesson, 1831) & RFA & Traumatogenic & VU & 4.1 & $\mathrm{r} 1,5$ \\
\hline \multicolumn{6}{|l|}{ Order Lamniformes } \\
\hline \multicolumn{6}{|l|}{ Family Myliobatidae } \\
\hline \multicolumn{6}{|l|}{ Genus Aetobatus } \\
\hline Aetobatus narinari (Euphrasen, 1790) & BRA & Traumatogenic & NT & 4.2 & r2 \\
\hline
\end{tabular}




\begin{tabular}{|c|c|c|c|c|c|}
\hline $\begin{array}{l}\text { Subclass, Order, Family, Genus, and } \\
\text { species }\end{array}$ & $\begin{array}{l}\text { Habitat } \\
\text { types }\end{array}$ & Threat to humans & $\begin{array}{l}\text { IUCN } \\
\text { status }\end{array}$ & $\begin{array}{l}\text { Trophic } \\
\text { level }\end{array}$ & $\begin{array}{l}\text { Other } \\
\text { remarks }\end{array}$ \\
\hline \multicolumn{6}{|l|}{ Genus Aetomylaeus } \\
\hline Aetomylaeus maculatus (Gray, 1834) & BRA & Traumatogenic & EN & 3.8 & r2 \\
\hline Aetomylaeus vespertilio (Bleeker, 1852) & BEP & & EN & 3.8 & r2 \\
\hline \multicolumn{6}{|l|}{ Family Alopiidae } \\
\hline \multicolumn{6}{|l|}{ Genus Alopias } \\
\hline Alopias vulpinus (Bonnaterre, 1788) & PEL & & VU & 4.5 & $\mathrm{r} 1, \mathrm{r} 6$ \\
\hline \multicolumn{6}{|l|}{ Family Lamnidae } \\
\hline \multicolumn{6}{|l|}{ Genus Isurus } \\
\hline Isurus oxyrinchus Rafinesque, 1810 & PEL & Traumatogenic & NT & 4.5 & r2 \\
\hline \multicolumn{6}{|l|}{ Order Carcharhiniformes } \\
\hline \multicolumn{6}{|l|}{ Family Scyliorhinidae } \\
\hline \multicolumn{6}{|l|}{ Genus Atelomycterus } \\
\hline $\begin{array}{l}\text { Atelomycterus marmoratus (Anonymous } \\
\text { [Bennett], 1830) }\end{array}$ & RFA & & NT & 4.1 & $\mathrm{r} 1$ \\
\hline \multicolumn{6}{|l|}{ Genus Cephaloscyllium } \\
\hline $\begin{array}{l}\text { Cephaloscyllium isabellum (Bonnaterre, } \\
1788 \text { ) }\end{array}$ & RFA & & LC & 4.2 & r2 \\
\hline $\begin{array}{l}\text { Cephaloscyllium umbratile Jordan \& } \\
\text { Fowler, } 1903\end{array}$ & RFA & & & 4.5 & r4 \\
\hline \multicolumn{6}{|l|}{ Family Triakidae } \\
\hline \multicolumn{6}{|l|}{ Genus Mustelus } \\
\hline Mustelus griseus Pietschmann, 1908 & DEM & & & 3.5 & r3 \\
\hline \multicolumn{6}{|l|}{ Family Carcharhinidae } \\
\hline \multicolumn{6}{|l|}{ Genus Carcharhinus } \\
\hline $\begin{array}{l}\text { Carcharhinus albimarginatus (Rüppell, } \\
\text { 1837) }\end{array}$ & RFA & Traumatogenic & VU & 4.2 & $\mathrm{r} 1$ \\
\hline $\begin{array}{l}\text { Carcharhinus amblyrhynchoides (Whitley, } \\
\text { 1934) }\end{array}$ & PN & Traumatogenic & NT & 4.2 & $\mathrm{r} 1, \mathrm{r} 7$ \\
\hline $\begin{array}{l}\text { Carcharhinus falciformis (Müller \& Henle, } \\
\text { 1839) }\end{array}$ & RFA & Traumatogenic & VU & 4.5 & $\mathrm{r} 1$ \\
\hline $\begin{array}{l}\text { Carcharhinus limbatus (Müller \& Henle, } \\
\text { 1839) }\end{array}$ & BRA & Traumatogenic & NT & 4.4 & r2 \\
\hline
\end{tabular}




\begin{tabular}{|c|c|c|c|c|c|}
\hline $\begin{array}{l}\text { Subclass, Order, Family, Genus, and } \\
\text { species }\end{array}$ & $\begin{array}{l}\text { Habitat } \\
\text { types }\end{array}$ & Threat to humans & $\begin{array}{l}\text { IUCN } \\
\text { status }\end{array}$ & $\begin{array}{l}\text { Trophic } \\
\text { level }\end{array}$ & $\begin{array}{l}\text { Other } \\
\text { remarks }\end{array}$ \\
\hline Carcharhinus longimanus (Poey, 1861) & PEL & Traumatogenic & VU & 4.2 & $\mathrm{r} 1$ \\
\hline \multicolumn{6}{|l|}{ Genus Galeocerdo } \\
\hline $\begin{array}{l}\text { Galeocerdo cuvier (Péron \& Lesueur, } \\
\text { 1822) }\end{array}$ & BRA & Traumatogenic & NT & 4.5 & $\mathrm{r} 1$ \\
\hline \multicolumn{6}{|l|}{ Genus Glyphis } \\
\hline Glyphis gangeticus (Müller \& Henle, 1839) & $\begin{array}{l}\text { DEM; } \\
\text { AMP }\end{array}$ & Traumatogenic & CE & 4.2 & $\mathrm{r} 1$ \\
\hline \multicolumn{6}{|l|}{ Genus Hemigaleus } \\
\hline Hemigaleus microstoma Bleeker, 1852 & DEM & & VU & 4.2 & r4 \\
\hline \multicolumn{6}{|l|}{ Genus Prionace } \\
\hline Prionace glauca (Linnaeus, 1758) & BRA & Traumatogenic & NT & 4.4 & r1 \\
\hline \multicolumn{6}{|l|}{ Genus Rhizoprionodon } \\
\hline Rhizoprionodon acutus (Rüppell, 1837) & $\begin{array}{l}\text { BEP; } \\
\text { AMP }\end{array}$ & & LC & 4.3 & r2 \\
\hline \multicolumn{6}{|l|}{ Genus Scoliodon } \\
\hline Scoliodon laticaudus Müller \& Henle, 1838 & BRA & & NT & 3.8 & $\mathrm{r} 1, \mathrm{r} 7$ \\
\hline \multicolumn{6}{|l|}{ Genus Triaenodon } \\
\hline Triaenodon obesus (Rüppell, 1837) & RFA & Traumatogenic & NT & 4.2 & r1 \\
\hline \multicolumn{6}{|l|}{ Family Sphyrnidae } \\
\hline \multicolumn{6}{|l|}{ Genus Sphyrna } \\
\hline Sphyrna lewini (Griffith \& Smith, 1834) & BRA & Other & VU & 4.1 & r1 \\
\hline \multicolumn{6}{|l|}{ Order Hexanchiformes } \\
\hline \multicolumn{6}{|l|}{ Family Hexanchidae } \\
\hline \multicolumn{6}{|l|}{ Genus Hexanchus } \\
\hline Hexanchus griseus (Bonnaterre, 1788) & BAD & Poisonous to eat & NT & 4.5 & $\mathrm{r} 1, \mathrm{r} 7$ \\
\hline Hexanchus nakamurai Teng, 1962 & BAD & & & 4.2 & $\mathrm{r} 1$ \\
\hline \multicolumn{6}{|l|}{ Genus Notorynchus } \\
\hline Notorynchus cepedianus (Péron, 1807) & BRA & Traumatogenic & LR & 4.7 & r4 \\
\hline \multicolumn{6}{|l|}{ Genus Myripristis } \\
\hline Myripristis botche Cuvier, 1829 & RFA & & LC & 4.0 & $\mathrm{r} 1$ \\
\hline
\end{tabular}




\begin{tabular}{|c|c|c|c|c|c|}
\hline $\begin{array}{l}\text { Subclass, Order, Family, Genus, and } \\
\text { species }\end{array}$ & $\begin{array}{l}\text { Habitat } \\
\text { types }\end{array}$ & Threat to humans & $\begin{array}{l}\text { IUCN } \\
\text { status }\end{array}$ & $\begin{array}{l}\text { Trophic } \\
\text { level }\end{array}$ & $\begin{array}{l}\text { Other } \\
\text { remarks }\end{array}$ \\
\hline $\begin{array}{l}\text { Myripristis chryseres Jordan \& Evermann, } \\
1903\end{array}$ & BEP & & LC & 4.0 & r2 \\
\hline Myripristis kuntee Valenciennes, 1831 & RFA & & LC & 3.4 & r6,r8 \\
\hline Myripristis murdjan (Forsskål, 1775) & RFA & $\begin{array}{l}\text { Reports of ciguatera } \\
\text { poisoning }\end{array}$ & LC & 3.4 & $\mathrm{r} 1, \mathrm{r} 7$ \\
\hline Myripristis pralinia Cuvier, 1829 & RFA & & LC & 3.5 & $\mathrm{r} 1$ \\
\hline Myripristis violacea Bleeker, 1851 & RFA & & LC & 3.5 & r4 \\
\hline Myripristis vittata Valenciennes, 1831 & RFA & & LC & 3.8 & r3 \\
\hline $\begin{array}{l}\text { *Myripristis robusta Randall \& Greenfield, } \\
1996\end{array}$ & DEM & & & & r11 \\
\hline \multicolumn{6}{|l|}{ Genus Neoniphon } \\
\hline $\begin{array}{l}\text { Neoniphon argenteus (Valenciennes, } \\
\text { 1831) }\end{array}$ & RFA & & LC & 4.0 & r3 \\
\hline $\begin{array}{l}\text { Neoniphon opercularis (Valenciennes, } \\
\text { 1831) }\end{array}$ & RFA & Venomous & LC & 3.6 & $\mathrm{r} 1, \mathrm{r} 6$ \\
\hline Neoniphon sammara (Forsskål, 1775) & RFA & Venomous & LC & 3.6 & $r 1, r 6, r 8, r 11$ \\
\hline \multicolumn{6}{|l|}{ Genus Ostichthys } \\
\hline Ostichthys kaianus (Günther, 1880) & BAD & & LC & 4.0 & r3 \\
\hline Ostichthys sheni Chen, Shao \& Mok, 1990 & DEM & & & 3.7 & r3 \\
\hline \multicolumn{6}{|l|}{ Genus Sargocentron } \\
\hline Sargocentron spiniferum (Forsskål, 1775) & RFA & $\begin{array}{l}\text { Reports of ciguatera } \\
\text { poisoning }\end{array}$ & LC & 3.6 & $\mathrm{r} 1$ \\
\hline Sargocentron violaceum (Bleeker, 1853) & RFA & Venomous & LC & 3.6 & $\mathrm{r} 1$ \\
\hline $\begin{array}{l}\text { Sargocentron caudimaculatum (Rüppell, } \\
1838 \text { ) }\end{array}$ & RFA & Venomous & LC & 3.9 & $\mathrm{r} 4, \mathrm{r} 6, \mathrm{r} 11$ \\
\hline Sargocentron cornutum (Bleeker, 1854) & BRA & Venomous & LC & 3.6 & r4 \\
\hline Sargocentron diadema (Lacepède, 1802) & RFA & Venomous & LC & 3.4 & r1 \\
\hline $\begin{array}{l}\text { Sargocentron ensifer (Jordan \& } \\
\text { Evermann, 1903) }\end{array}$ & RFA & & LC & 4.0 & $\mathrm{r} 1, \mathrm{r} 7$ \\
\hline $\begin{array}{l}\text { Sargocentron punctatissimum (Cuvier, } \\
\text { 1829) }\end{array}$ & RFA & & LC & 3.4 & $\mathrm{r} 1, \mathrm{r} 7$ \\
\hline Sargocentron rubrum (Forsskål, 1775) & RFA & Venomous & LC & 3.6 & $\mathrm{r} 4, \mathrm{r} 7$ \\
\hline
\end{tabular}




\begin{tabular}{|c|c|c|c|c|c|}
\hline $\begin{array}{l}\text { Subclass, Order, Family, Genus, and } \\
\text { species }\end{array}$ & $\begin{array}{l}\text { Habitat } \\
\text { types }\end{array}$ & Threat to humans & $\begin{array}{l}\text { IUCN } \\
\text { status }\end{array}$ & $\begin{array}{l}\text { Trophic } \\
\text { level }\end{array}$ & $\begin{array}{l}\text { Other } \\
\text { remarks }\end{array}$ \\
\hline Sargocentron spiniferum (Forsskål, 1775) & RFA & $\begin{array}{l}\text { Reports of ciguatera } \\
\text { poisoning }\end{array}$ & LC & 3.6 & r4 \\
\hline Sargocentron tiere (Cuvier, 1829) & RFA & Venomous & LC & 4.2 & r3 \\
\hline Sargocentron violaceum (Bleeker, 1853) & RFA & Venomous & LC & 3.6 & r4 \\
\hline $\begin{array}{l}\text { Sargocentron melanospilos (Bleeker, } \\
1858 \text { ) }\end{array}$ & RFA & Venomous & LC & 4.0 & r9,r11 \\
\hline Sargocentron microstoma (Günther, 1859) & RFA & Venomous & LC & 3.6 & $r 10, r 11$ \\
\hline \multicolumn{6}{|l|}{ Order Squatiniformes } \\
\hline \multicolumn{6}{|l|}{ Family Squalidae } \\
\hline \multicolumn{6}{|l|}{ Genus Centrophorus } \\
\hline Centrophorus tessellatus Garman, 1906 & BEP & & & 4.3 & $\mathrm{r} 1$ \\
\hline \multicolumn{6}{|l|}{ Genus Squalus } \\
\hline Squalus brevirostris Tanaka, 1917 & DEM & & & 4.2 & $\mathrm{r} 1$ \\
\hline Squalus megalops (Macleay, 1881) & DEM & & & 4.3 & r2 \\
\hline \multicolumn{6}{|l|}{ Order Rajiformes } \\
\hline \multicolumn{6}{|l|}{ Family Rhinobatidae } \\
\hline \multicolumn{6}{|l|}{ Genus Rhina } \\
\hline $\begin{array}{l}\text { Rhina ancylostoma Bloch \& Schneider, } \\
1801\end{array}$ & RFA & Other & VU & 3.6 & $\mathrm{r} 1$ \\
\hline \multicolumn{6}{|l|}{ Genus Rhynchobatus } \\
\hline Rhynchobatus djiddensis (Forsskål, 1775) & BRA & & VU & 3.6 & $\mathrm{r} 1$ \\
\hline \multicolumn{6}{|l|}{ Order Myliobatiformes } \\
\hline \multicolumn{6}{|l|}{ Family Dasyatidae } \\
\hline \multicolumn{6}{|l|}{ Genus Hemitrygon } \\
\hline $\begin{array}{l}\text { Hemitrygon bennettii (Müller \& Henle, } \\
\text { 1841) }\end{array}$ & DEM & & & 4.5 & r2 \\
\hline Hemitrygon sinensis (Steindachner, 1892) & DEM & & & 3.7 & $\mathrm{r} 2$ \\
\hline \multicolumn{6}{|l|}{ Genus Neotrygon } \\
\hline Neotrygon kuhlii (Müller \& Henle, 1841) & RFA & Venomous & & 3.3 & r2 \\
\hline Genus Pteroplatytrygon & & & & & \\
\hline
\end{tabular}




\begin{tabular}{|c|c|c|c|c|c|}
\hline $\begin{array}{l}\text { Subclass, Order, Family, Genus, and } \\
\text { species }\end{array}$ & $\begin{array}{l}\text { Habitat } \\
\text { types }\end{array}$ & Threat to humans & $\begin{array}{l}\text { IUCN } \\
\text { status }\end{array}$ & $\begin{array}{l}\text { Trophic } \\
\text { level }\end{array}$ & $\begin{array}{l}\text { Other } \\
\text { remarks }\end{array}$ \\
\hline $\begin{array}{l}\text { Pteroplatytrygon violacea (Bonaparte, } \\
\text { 1832) }\end{array}$ & PEL & Venomous & LC & 4.4 & r1 \\
\hline \multicolumn{6}{|l|}{ Genus Taeniurops } \\
\hline Taeniurops meyeni (Müller \& Henle, 1841) & RFA & Venomous & VU & 4.2 & $\mathrm{r} 1$ \\
\hline \multicolumn{6}{|l|}{ Genus Urogymnus } \\
\hline $\begin{array}{l}\text { Urogymnus asperrimus (Bloch \& } \\
\text { Schneider, 1801) }\end{array}$ & BRA & Traumatogenic & VU & 3.5 & r2 \\
\hline \multicolumn{6}{|l|}{ Family Gymnuridae } \\
\hline \multicolumn{6}{|l|}{ Genus Gymnura } \\
\hline $\begin{array}{l}\text { Gymnura japonica (Temminck \& Schlegel, } \\
\text { 1850) }\end{array}$ & DEM & Venomous & & 3.8 & r2 \\
\hline \multicolumn{6}{|l|}{ Order Anguilliformes } \\
\hline \multicolumn{6}{|l|}{ Family Muraenidae } \\
\hline \multicolumn{6}{|l|}{ Genus Echidna } \\
\hline Echidna delicatula (Kaup, 1856) & RFA & & & 3.5 & $\mathrm{r} 1$ \\
\hline Echidna nebulosa (Ahl, 1789) & RFA & & LC & 4.0 & r1 \\
\hline Echidna polyzona (Richardson, 1845) & RFA & & LC & 3.5 & $\mathrm{r} 1$ \\
\hline \multicolumn{6}{|l|}{ Genus Gymnomuraena } \\
\hline Gymnomuraena zebra (Shaw, 1797) & RFA & & LC & 3.4 & $\mathrm{r} 1$ \\
\hline \multicolumn{6}{|l|}{ Genus Gymnothorax } \\
\hline Gymnothorax fimbriatus (Bennett, 1832) & BRA & & LC & 4.0 & $\mathrm{r} 1$ \\
\hline $\begin{array}{l}\text { Gymnothorax flavimarginatus (Rüppell, } \\
\text { 1830) }\end{array}$ & RFA & $\begin{array}{l}\text { Reports of ciguatera } \\
\text { poisoning }\end{array}$ & LC & 4.2 & $\mathrm{r} 1$ \\
\hline $\begin{array}{l}\text { Gymnothorax isingteena (Richardson, } \\
1845 \text { ) }\end{array}$ & RFA & & LC & 4.3 & $\mathrm{r} 4$ \\
\hline Gymnothorax meleagris (Shaw, 1795) & RFA & $\begin{array}{l}\text { Reports of ciguatera } \\
\text { poisoning }\end{array}$ & LC & 4.0 & $r 1, r 6, r 11$ \\
\hline Gymnothorax pictus (Ahl, 1789) & BRA & $\begin{array}{l}\text { Reports of ciguatera } \\
\text { poisoning }\end{array}$ & LC & 4.2 & $\mathrm{r} 1$ \\
\hline Gymnothorax prionodon Ogilby, 1895 & RFA & & LC & 4.2 & r3 \\
\hline $\begin{array}{l}\text { Gymnothorax pseudothyrsoideus (Bleeker, } \\
\text { 1853) }\end{array}$ & RFA & & LC & 3.7 & r4 \\
\hline
\end{tabular}




\begin{tabular}{|c|c|c|c|c|c|}
\hline $\begin{array}{l}\text { Subclass, Order, Family, Genus, and } \\
\text { species }\end{array}$ & $\begin{array}{l}\text { Habitat } \\
\text { types }\end{array}$ & Threat to humans & $\begin{array}{l}\text { IUCN } \\
\text { status }\end{array}$ & $\begin{array}{l}\text { Trophic } \\
\text { level }\end{array}$ & $\begin{array}{l}\text { Other } \\
\text { remarks }\end{array}$ \\
\hline Gymnothorax reevesii (Richardson, 1845) & RFA & & & 4.0 & r1 \\
\hline Gymnothorax richardsonii (Bleeker, 1852) & RFA & & LC & 3.8 & $\mathrm{r} 1$ \\
\hline $\begin{array}{l}\text { Gymnothorax rueppelliae (McClelland, } \\
\text { 1844) }\end{array}$ & BRA & & LC & 4.0 & $\mathrm{r} 1$ \\
\hline $\begin{array}{l}\text { Gymnothorax thyrsoideus (Richardson, } \\
1845 \text { ) }\end{array}$ & RFA & & LC & 4.0 & $\mathrm{r} 1$ \\
\hline Gymnothorax undulatus (Lacepède, 1803) & BRA & $\begin{array}{l}\text { Reports of ciguatera } \\
\text { poisoning }\end{array}$ & LC & 3.6 & $\mathrm{r} 1$ \\
\hline \multicolumn{6}{|l|}{ Genus Scuticaria } \\
\hline Scuticaria tigrina (Lesson, 1828) & RFA & & LC & 3.8 & $\mathrm{r} 1$ \\
\hline \multicolumn{6}{|l|}{ Family Ophichthyidae } \\
\hline \multicolumn{6}{|l|}{ Genus Myrichthys } \\
\hline Myrichthys colubrinus (Boddaert, 1781) & RFA & & & 3.6 & $\mathrm{r} 1$ \\
\hline Myrichthys maculosus (Cuvier, 1816) & RFA & & & 3.6 & $\mathrm{r} 1$ \\
\hline \multicolumn{6}{|l|}{ Genus Pisoodonophis } \\
\hline Pisoodonophis rubicundus & & & & 4.0 & $\mathrm{r} 1$ \\
\hline \multicolumn{6}{|l|}{ Order Clupeiformes } \\
\hline \multicolumn{6}{|l|}{ Family Clupeidae } \\
\hline \multicolumn{6}{|l|}{ Genus Amblygaster } \\
\hline Amblygaster clupeoides Bleeker, 1849 & RFA & & LC & 3.1 & $\mathrm{r} 1$ \\
\hline \multicolumn{6}{|l|}{ Genus Conger } \\
\hline Conger cinereus Rüppell, 1830 & RFA & & LC & 4.3 & $\mathrm{r} 1$ \\
\hline \multicolumn{6}{|l|}{ Order Gonorhynchiformes } \\
\hline \multicolumn{6}{|l|}{ Family Chanidae } \\
\hline \multicolumn{6}{|l|}{ Genusa Chanos } \\
\hline Chanos chanos (Forsskål, 1775) & BRA & & LC & 2.4 & $\mathrm{r} 1$ \\
\hline \multicolumn{6}{|l|}{ Family Centriscidae } \\
\hline \multicolumn{6}{|l|}{ Genus Aeoliscus } \\
\hline Aeoliscus strigatus (Günther, 1861) & RFA & & & 3.5 & r6 \\
\hline \multicolumn{6}{|l|}{ Order Stomiiformes } \\
\hline Family Sternoptychidae & & & & & \\
\hline
\end{tabular}




\begin{tabular}{|c|c|c|c|c|c|}
\hline $\begin{array}{l}\text { Subclass, Order, Family, Genus, and } \\
\text { species }\end{array}$ & $\begin{array}{l}\text { Habitat } \\
\text { types }\end{array}$ & Threat to humans & $\begin{array}{l}\text { IUCN } \\
\text { status }\end{array}$ & $\begin{array}{l}\text { Trophic } \\
\text { level }\end{array}$ & $\begin{array}{l}\text { Other } \\
\text { remarks }\end{array}$ \\
\hline \multicolumn{6}{|l|}{ Genus Sternoptyx } \\
\hline Sternoptyx diaphana Hermann, 1781 & BAP & & & 3.4 & $\mathrm{r} 1$ \\
\hline Sternoptyx obscura Garman, 1899 & BAP & & LC & 3.3 & r1 \\
\hline \multicolumn{6}{|l|}{ Family Phosichthyidae } \\
\hline \multicolumn{6}{|l|}{ Genus Polymetme } \\
\hline Polymetme corythaeola (Alcock, 1898) & BEP & & & 4.4 & r1 \\
\hline \multicolumn{6}{|l|}{ Order Aulopiformes } \\
\hline \multicolumn{6}{|l|}{ Family Synodontidae } \\
\hline \multicolumn{6}{|l|}{ Genus Saurida } \\
\hline Saurida gracilis (Quoy \& Gaimard, 1824) & RFA & & LC & 4.2 & r1 \\
\hline \multicolumn{6}{|l|}{ Genus Synodus } \\
\hline Synodus jaculum Russell \& Cressey, 1979 & RFA & & LC & 4.0 & r1 \\
\hline Synodus variegatus (Lacepède, 1803) & RFA & & LC & 4.2 & r1 \\
\hline \multicolumn{6}{|l|}{ Family Alepisauridae } \\
\hline \multicolumn{6}{|l|}{ Genus Alepisaurus } \\
\hline Alepisaurus ferox Lowe, 1833 & BAP & & LC & 4.0 & r1 \\
\hline \multicolumn{6}{|l|}{ Order Myctophiformes } \\
\hline \multicolumn{6}{|l|}{ Family Myctophidae } \\
\hline \multicolumn{6}{|l|}{ Genus Myctophum } \\
\hline Myctophum aurolaternatum Garman, 1899 & BAP & & LC & 3.5 & $\mathrm{r} 1$ \\
\hline \multicolumn{6}{|l|}{ Order Polymixiiformes } \\
\hline \multicolumn{6}{|l|}{ Family Polymixiidae } \\
\hline \multicolumn{6}{|l|}{ Genus Polymixia } \\
\hline Polymixia berndti Gilbert, 1905 & RFA & & LC & 4.0 & $\mathrm{r} 1$ \\
\hline \multicolumn{6}{|l|}{ Order Ophidiiformes } \\
\hline \multicolumn{6}{|l|}{ Family Carapidae } \\
\hline \multicolumn{6}{|l|}{ Genus Carapus } \\
\hline Carapus mourlani (Petit, 1934) & RFA & & & 3.6 & $\mathrm{r} 1$ \\
\hline \multicolumn{6}{|l|}{ Genus Encheliophis } \\
\hline Encheliophis homei (Richardson, 1846) & DEM & & & 3.7 & $\mathrm{r} 1$ \\
\hline
\end{tabular}




\begin{tabular}{|c|c|c|c|c|c|}
\hline $\begin{array}{l}\text { Subclass, Order, Family, Genus, and } \\
\text { species }\end{array}$ & $\begin{array}{l}\text { Habitat } \\
\text { types }\end{array}$ & Threat to humans & $\begin{array}{l}\text { IUCN } \\
\text { status }\end{array}$ & $\begin{array}{l}\text { Trophic } \\
\text { level }\end{array}$ & $\begin{array}{l}\text { Other } \\
\text { remarks }\end{array}$ \\
\hline Encheliophis boraborensis (Kaup, 1856) & DEM & & & 3.4 & $\mathrm{r} 1$ \\
\hline \multicolumn{6}{|l|}{ Family Bythitidae } \\
\hline \multicolumn{6}{|l|}{ Genus Dinematichthys } \\
\hline $\begin{array}{l}\text { Dinematichthys iluocoeteoides Bleeker, } \\
1855\end{array}$ & RFA & & & 2.6 & $\mathrm{r} 1$ \\
\hline \multicolumn{6}{|l|}{ Order Lophiiformes } \\
\hline \multicolumn{6}{|l|}{ Family Antennaridae } \\
\hline \multicolumn{6}{|l|}{ Genus Antennatus } \\
\hline Antennatus dorehensis (Bleeker, 1859) & RFA & & & 4.3 & $\mathrm{r} 1$ \\
\hline \multicolumn{6}{|l|}{ Family Chaunacidae } \\
\hline \multicolumn{6}{|l|}{ Genus Chaunax } \\
\hline Chaunax fimbriatus Hilgendorf, 1879 & BAD & & & 3.9 & $\mathrm{r} 1$ \\
\hline \multicolumn{6}{|l|}{ Family Ogcocephalidae } \\
\hline \multicolumn{6}{|l|}{ Genus Halicmetus } \\
\hline $\begin{array}{l}\text { Halicmetus reticulatus Smith \& Radcliffe, } \\
1912\end{array}$ & BAD & & & 3.3 & $\mathrm{r} 1$ \\
\hline \multicolumn{6}{|l|}{ Genus Halieutaea } \\
\hline $\begin{array}{l}\text { Halieutaea indica Annandale \& Jenkins, } \\
1910\end{array}$ & DEM & & & 3.4 & r1 \\
\hline \multicolumn{6}{|l|}{ Order Mugiliformes } \\
\hline \multicolumn{6}{|l|}{ Family Mugilidae } \\
\hline \multicolumn{6}{|l|}{ Genus Crenimugil } \\
\hline Crenimugil crenilabis (Forsskål, 1775) & BRA & & LC & 2.3 & $\mathrm{r} 1$ \\
\hline \multicolumn{6}{|l|}{ Genus Ellochelon } \\
\hline $\begin{array}{l}\text { Ellochelon vaigiensis (Quoy \& Gaimard, } \\
\text { 1825) }\end{array}$ & RFA; & & LC & 2.2 & $\mathrm{r} 1$ \\
\hline \multicolumn{6}{|l|}{ Genus Moolgarda } \\
\hline Crenimugil seheli (Forsskål, 1775) & RFA; & & & 2.3 & r1 \\
\hline \multicolumn{6}{|l|}{ Genus Oedalechilus } \\
\hline Plicomugil labiosus (Valenciennes, 1836) & RFA & & & 2.1 & $\mathrm{r} 1$ \\
\hline Order Atheriniformes & & & & & \\
\hline
\end{tabular}




\begin{tabular}{|c|c|c|c|c|c|}
\hline $\begin{array}{l}\text { Subclass, Order, Family, Genus, and } \\
\text { species }\end{array}$ & $\begin{array}{l}\text { Habitat } \\
\text { types }\end{array}$ & Threat to humans & $\begin{array}{l}\text { IUCN } \\
\text { status }\end{array}$ & $\begin{array}{l}\text { Trophic } \\
\text { level }\end{array}$ & $\begin{array}{l}\text { Other } \\
\text { remarks }\end{array}$ \\
\hline \multicolumn{6}{|l|}{ Family Atherinidae } \\
\hline \multicolumn{6}{|l|}{ Genus Atherinomorus } \\
\hline Atherinomorus lacunosus (Forster, 1801) & RFA; & & & 3.3 & $\mathrm{r} 1$ \\
\hline \multicolumn{6}{|l|}{ Family Exocoetidae } \\
\hline \multicolumn{6}{|l|}{ Genus Cheilopogon } \\
\hline Cheilopogon arcticeps (Günther, 1866) & PN & & & 3.6 & r1 \\
\hline Cheilopogon atrisignis (Jenkins, 1903) & PEL & & & 3.7 & r1 \\
\hline $\begin{array}{l}\text { Cheilopogon cyanopterus (Valenciennes, } \\
1847 \text { ) }\end{array}$ & PEL & & LC & 3.3 & $\mathrm{r} 1$ \\
\hline Cheilopogon katoptron (Bleeker, 1865) & PN & & & 3.5 & $\mathrm{r} 1$ \\
\hline $\begin{array}{l}\text { Cheilopogon pinnatibarbatus } \\
\text { pinnatibarbatus (Bennett, 1831) }\end{array}$ & PN & & LC & 4.0 & $\mathrm{r} 1$ \\
\hline $\begin{array}{l}\text { Cheilopogon spilopterus (Valenciennes, } \\
1847 \text { ) }\end{array}$ & PN & & & 4.2 & $\mathrm{r} 1$ \\
\hline \multicolumn{6}{|l|}{ Genus Cypselurus } \\
\hline Cypselurus oligolepis (Bleeker, 1865) & PN & & & 4.0 & $\mathrm{r} 1$ \\
\hline $\begin{array}{l}\text { Cypselurus poecilopterus (Valenciennes, } \\
\text { 1847) }\end{array}$ & PN; OD & & & 3.4 & $\mathrm{r} 1$ \\
\hline \multicolumn{6}{|l|}{ Genus Exocoetus } \\
\hline Exocoetus volitans Linnaeus, 1758 & $\mathrm{PN} ; \mathrm{OD}$ & & LC & 3.0 & $r 1, r 6$ \\
\hline \multicolumn{6}{|l|}{ Genus Hirundichthys } \\
\hline $\begin{array}{l}\text { Hirundichthys oxycephalus (Bleeker, } \\
\text { 1853) }\end{array}$ & $\mathrm{PN} ; \mathrm{OD}$ & & & 3.0 & $\mathrm{r} 1$ \\
\hline $\begin{array}{l}\text { Hirundichthys speculiger (Valenciennes, } \\
\text { 1847) }\end{array}$ & PEL; OD & & LC & 3.0 & $\mathrm{r} 1$ \\
\hline \multicolumn{6}{|l|}{ Genus Parexocoetus } \\
\hline $\begin{array}{l}\text { Parexocoetus brachypterus (Richardson, } \\
\text { 1846) }\end{array}$ & PN; OD & & & 3.4 & $\mathrm{r} 1$ \\
\hline \multicolumn{6}{|l|}{ Family Hemiramphidae } \\
\hline \multicolumn{6}{|l|}{ Genus Euleptorhamphus } \\
\hline $\begin{array}{l}\text { Euleptorhamphus viridis (van Hasselt, } \\
\text { 1823) }\end{array}$ & PEL; OD & & & 3.4 & $r 1, r 8$ \\
\hline
\end{tabular}




\begin{tabular}{|c|c|c|c|c|c|}
\hline $\begin{array}{l}\text { Subclass, Order, Family, Genus, and } \\
\text { species }\end{array}$ & $\begin{array}{l}\text { Habitat } \\
\text { types }\end{array}$ & Threat to humans & $\begin{array}{l}\text { IUCN } \\
\text { status }\end{array}$ & $\begin{array}{l}\text { Trophic } \\
\text { level }\end{array}$ & $\begin{array}{l}\text { Other } \\
\text { remarks }\end{array}$ \\
\hline \multicolumn{6}{|l|}{ Genus Hyporhamphus } \\
\hline $\begin{array}{l}\text { Hyporhamphus dussumieri (Valenciennes, } \\
1847 \text { ) }\end{array}$ & RFA & & & 3.5 & $\mathrm{r} 1$ \\
\hline $\begin{array}{l}\text { Hyporhamphus gernaerti (Valenciennes, } \\
1847 \text { ) }\end{array}$ & PN & & & 3.0 & $\mathrm{r} 1$ \\
\hline \multicolumn{6}{|l|}{ Family Belonidae } \\
\hline \multicolumn{6}{|l|}{ Genus Ablennes } \\
\hline Ablennes hians (Valenciennes, 1846) & RFA; OD & & LC & 4.5 & $\mathrm{r} 1$ \\
\hline \multicolumn{6}{|l|}{ Genus Platybelone } \\
\hline $\begin{array}{l}\text { Platybelone argalus argalus (Lesueur, } \\
\text { 1821) }\end{array}$ & RFA & & LC & 4.5 & $\mathrm{r} 1$ \\
\hline \multicolumn{6}{|l|}{ Genus Tylosurus } \\
\hline Tylosurus acus acus (Lacepède, 1803) & RFA & Traumatogenic & LC & 4.5 & r1 \\
\hline \multicolumn{6}{|l|}{ Order Gasterosteiformes } \\
\hline \multicolumn{6}{|l|}{ Family Pegasidae } \\
\hline \multicolumn{6}{|l|}{ Genus Pegasus } \\
\hline Pegasus laternarius Cuvier, 1816 & DEM & & & 3.3 & r1 \\
\hline \multicolumn{6}{|l|}{ Family Aulostomidae } \\
\hline \multicolumn{6}{|l|}{ Genus Aulostomus } \\
\hline Aulostomus chinensis (Linnaeus, 1766) & RFA & & LC & 4.2 & $\mathrm{r} 1$ \\
\hline \multicolumn{6}{|l|}{ Genus Centriscus } \\
\hline Centriscus scutatus Linnaeus, 1758 & RFA & & LC & 3.3 & $\mathrm{r} 1$ \\
\hline \multicolumn{6}{|l|}{ Family Fistulariidae } \\
\hline \multicolumn{6}{|l|}{ Genus Fistularia } \\
\hline Fistularia petimba Lacepède, 1803 & RFA & & LC & 4.4 & $r 1, r 8$ \\
\hline \multicolumn{6}{|l|}{ Order synbranchiformes } \\
\hline \multicolumn{6}{|l|}{ Family Syngnathidae } \\
\hline \multicolumn{6}{|l|}{ Genus Corythoichthys } \\
\hline $\begin{array}{l}\text { Corythoichthys flavofasciatus (Rüppell, } \\
\text { 1838) }\end{array}$ & RFA & & LC & 3.6 & $r 1, r 6$ \\
\hline Genus Doryrhamphus & & & & & \\
\hline
\end{tabular}




\begin{tabular}{|c|c|c|c|c|c|}
\hline $\begin{array}{l}\text { Subclass, Order, Family, Genus, and } \\
\text { species }\end{array}$ & $\begin{array}{l}\text { Habitat } \\
\text { types }\end{array}$ & Threat to humans & $\begin{array}{l}\text { IUCN } \\
\text { status }\end{array}$ & $\begin{array}{l}\text { Trophic } \\
\text { level }\end{array}$ & $\begin{array}{l}\text { Other } \\
\text { remarks }\end{array}$ \\
\hline $\begin{array}{l}\text { Doryrhamphus excisus excisus Kaup, } \\
1856\end{array}$ & RFA & & LC & 3.5 & $\mathrm{r} 1$ \\
\hline \multicolumn{6}{|l|}{ Genus Trachyrhamphus } \\
\hline $\begin{array}{l}\text { Trachyrhamphus serratus (Temminck \& } \\
\text { Schlegel, 1850) }\end{array}$ & RFA & & & 3.7 & $\mathrm{r} 1$ \\
\hline \multicolumn{6}{|l|}{ Order Scorpaeniformes } \\
\hline \multicolumn{6}{|l|}{ Family Dactylopteridae } \\
\hline \multicolumn{6}{|l|}{ Genus Dactyloptena } \\
\hline Dactyloptena orientalis (Cuvier, 1829) & RFA & & LC & 3.7 & $\mathrm{r} 1$ \\
\hline \multicolumn{6}{|l|}{ Family Scorpaenidae } \\
\hline \multicolumn{6}{|l|}{ Genus Dendrochirus } \\
\hline $\begin{array}{l}\text { Dendrochirus bellus (Jordan \& Hubbs, } \\
\text { 1925) }\end{array}$ & DEM & & LC & 3.8 & $\mathrm{r} 1$ \\
\hline Dendrochirus zebra (Cuvier, 1829) & RFA & Venomous & LC & 4.0 & $\mathrm{r} 1$ \\
\hline \multicolumn{6}{|l|}{ Genus Parapterois } \\
\hline Parapterois heterura (Bleeker, 1856) & DEM & Venomous & LC & 3.9 & $\mathrm{r} 1$ \\
\hline \multicolumn{6}{|l|}{ Genus Parascorpaena } \\
\hline Parascorpaena aurita (Rüppell, 1838) & RFA & & LC & 3.7 & $\mathrm{r} 1$ \\
\hline Parascorpaena picta (Cuvier, 1829) & RFA & & LC & 3.7 & $\mathrm{r} 1$ \\
\hline \multicolumn{6}{|l|}{ Genus Pterois } \\
\hline Pterois radiata Cuvier, 1829 & RFA & Venomous & LC & 3.6 & $\mathrm{r} 1, \mathrm{r} 6$ \\
\hline Pterois volitans (Linnaeus, 1758) & RFA & Venomous & LC & 4.4 & $\mathrm{r} 1, \mathrm{r} 6$ \\
\hline \multicolumn{6}{|l|}{ Genus Scorpaena } \\
\hline Scorpaena hatizyoensis Matsubara, 1943 & DEM & & LC & 3.7 & r1 \\
\hline $\begin{array}{l}\text { Scorpaena neglecta Temminck \& } \\
\text { Schlegel, } 1843\end{array}$ & DEM & & & 3.8 & r1 \\
\hline \multicolumn{6}{|l|}{ Genus Scorpaenodes } \\
\hline $\begin{array}{l}\text { Scorpaenodes guamensis (Quoy \& } \\
\text { Gaimard, 1824) }\end{array}$ & RFA & Venomous & LC & 3.4 & $\mathrm{r} 1$ \\
\hline $\begin{array}{l}\text { Scorpaenodes scaber (Ramsay \& Ogilby, } \\
1886 \text { ) }\end{array}$ & RFA & Venomous & & 3.6 & $\mathrm{r} 1$ \\
\hline
\end{tabular}




\begin{tabular}{|c|c|c|c|c|c|}
\hline $\begin{array}{l}\text { Subclass, Order, Family, Genus, and } \\
\text { species }\end{array}$ & $\begin{array}{l}\text { Habitat } \\
\text { types }\end{array}$ & Threat to humans & $\begin{array}{l}\text { IUCN } \\
\text { status }\end{array}$ & $\begin{array}{l}\text { Trophic } \\
\text { level }\end{array}$ & $\begin{array}{l}\text { Other } \\
\text { remarks }\end{array}$ \\
\hline \multicolumn{6}{|l|}{ Genus Scorpaenopsis } \\
\hline Scorpaenopsis cirrosa (Thunberg,1793) & DEM & Venomous & & 3.5 & $\mathrm{r} 1$ \\
\hline \multicolumn{6}{|l|}{ Genus Sebastapistes } \\
\hline $\begin{array}{l}\text { *Sebastapistes cyanostigma (Bleeker, } \\
\text { 1856) }\end{array}$ & RFA & & & 3.8 & $\mathrm{r} 11$ \\
\hline Sebastapistes nuchalis (Günther, 1874) & DEM & & & 3.8 & $\mathrm{r} 1$ \\
\hline \multicolumn{6}{|l|}{ Genus Synanceia } \\
\hline $\begin{array}{l}\text { Synanceia verrucosa Bloch \& Schneider, } \\
1801\end{array}$ & RFA & Venomous & LC & 4.2 & $\mathrm{r} 1$ \\
\hline \multicolumn{6}{|l|}{ Genus Vespicula } \\
\hline Vespicula trachinoides (Cuvier, 1829) & DEM & & & 3.1 & r1 \\
\hline \multicolumn{6}{|l|}{ Order perciformes } \\
\hline \multicolumn{6}{|l|}{ Family Serranidae } \\
\hline \multicolumn{6}{|l|}{ Genus Aethaloperca } \\
\hline Aethaloperca rogaa (Forsskål, 1775) & RFA & & LC & 4.2 & $\mathrm{r} 1, \mathrm{r} 11$ \\
\hline \multicolumn{6}{|l|}{ Genus Anyperodon } \\
\hline $\begin{array}{l}\text { Anyperodon leucogrammicus } \\
\text { (Valenciennes, 1828) }\end{array}$ & RFA & & LC & 3.9 & $\mathrm{r} 1$ \\
\hline \multicolumn{6}{|l|}{ Genus Cephalopholis } \\
\hline Cephalopholis argus Schneider, 1801 & RFA & $\begin{array}{l}\text { Reports of ciguatera } \\
\text { poisoning }\end{array}$ & LC & 4.5 & $\mathrm{r} 1, \mathrm{r} 6, \mathrm{r} 11$ \\
\hline $\begin{array}{l}\text { Cephalopholis aurantia (Valenciennes, } \\
\text { 1828) }\end{array}$ & RFA & & LC & 4.0 & $\mathrm{r} 1$ \\
\hline Cephalopholis formosa (Shaw, 1812) & RFA & & LC & 4.1 & $\mathrm{r} 1$ \\
\hline $\begin{array}{l}\text { Cephalopholis leopardus (Lacepède, } \\
\text { 1801) }\end{array}$ & RFA & & LC & 4.0 & $\mathrm{r} 1$ \\
\hline Cephalopholis miniata (Forsskål, 1775) & RFA & & LC & 4.3 & $\mathrm{r} 1$ \\
\hline $\begin{array}{l}\text { Cephalopholis sonnerati (Valenciennes, } \\
\text { 1828) }\end{array}$ & RFA & & LC & 3.8 & r1 \\
\hline Cephalopholis urodeta (Forster, 1801) & RFA & & LC & 4.0 & $\mathrm{r} 1, \mathrm{r} 6, \mathrm{r} 11$ \\
\hline Genus Epinephelus & & & & & \\
\hline
\end{tabular}




\begin{tabular}{|c|c|c|c|c|c|}
\hline $\begin{array}{l}\text { Subclass, Order, Family, Genus, and } \\
\text { species }\end{array}$ & $\begin{array}{l}\text { Habitat } \\
\text { types }\end{array}$ & Threat to humans & $\begin{array}{l}\text { IUCN } \\
\text { status }\end{array}$ & $\begin{array}{l}\text { Trophic } \\
\text { level }\end{array}$ & $\begin{array}{l}\text { Other } \\
\text { remarks }\end{array}$ \\
\hline $\begin{array}{l}\text { Epinephelus akaara (Temminck \& } \\
\text { Schlegel, 1842) }\end{array}$ & RFA & & EN & 4.0 & $\mathrm{r} 1$ \\
\hline Epinephelus areolatus (Forsskål, 1775) & RFA & & LC & 3.7 & $\mathrm{r} 1$ \\
\hline $\begin{array}{l}\text { Epinephelus awoara (Temminck \& } \\
\text { Schlegel, 1842) }\end{array}$ & RFA & & & 3.6 & $\mathrm{r} 1$ \\
\hline $\begin{array}{l}\text { Epinephelus chlorostigma (Valenciennes, } \\
\text { 1828) }\end{array}$ & RFA & & LC & 4.0 & r1 \\
\hline Epinephelus coioides (Hamilton, 1822) & RFA & & LC & 4.0 & $\mathrm{r} 1$ \\
\hline $\begin{array}{l}\text { Epinephelus cyanopodus (Richardson, } \\
\text { 1846) }\end{array}$ & RFA & $\begin{array}{l}\text { Reports of ciguatera } \\
\text { poisoning }\end{array}$ & LC & 4.1 & $\mathrm{r} 4, \mathrm{r} 6$ \\
\hline Epinephelus fasciatus (Forsskål, 1775) & RFA & $\begin{array}{l}\text { Reports of ciguatera } \\
\text { poisoning }\end{array}$ & LC & 3.7 & $\mathrm{r} 1, \mathrm{r} 7, \mathrm{r} 11$ \\
\hline $\begin{array}{l}\text { Epinephelus fuscoguttatus (Forsskål, } \\
\text { 1775) }\end{array}$ & RFA & $\begin{array}{l}\text { Reports of ciguatera } \\
\text { poisoning }\end{array}$ & VU & 4.1 & $\mathrm{r} 1$ \\
\hline Epinephelus heniochus Fowler, 1904 & DEM & & LC & 3.8 & $\mathrm{r} 1$ \\
\hline Epinephelus hexagonatus (Forster, 1801) & RFA & & LC & 4.1 & $\mathrm{r} 1, \mathrm{r} 11$ \\
\hline $\begin{array}{l}\text { Epinephelus latifasciatus (Temminck \& } \\
\text { Schlegel, 1842) }\end{array}$ & DEM & & LC & 4.0 & $\mathrm{r} 1$ \\
\hline Epinephelus lanceolatus (Bloch, 1790) & RFA & Traumatogenic & & 4.0 & $\mathrm{r} 1$ \\
\hline Epinephelus longispinis (Kner, 1864) & RFA & & LC & 4.2 & $\mathrm{r} 1$ \\
\hline Epinephelus maculatus (Bloch, 1790) & RFA & $\begin{array}{l}\text { Reports of ciguatera } \\
\text { poisoning }\end{array}$ & LC & 4.0 & r5 \\
\hline Epinephelus merra Bloch, 1793 & RFA & $\begin{array}{l}\text { Reports of ciguatera } \\
\text { poisoning }\end{array}$ & LC & 3.8 & $r 1, r 6, r 8, r 11$ \\
\hline $\begin{array}{l}\text { Epinephelus morrhua (Valenciennes, } \\
\text { 1833) }\end{array}$ & RFA & $\begin{array}{l}\text { Reports of ciguatera } \\
\text { poisoning }\end{array}$ & LC & 4.0 & $\mathrm{r} 1$ \\
\hline $\begin{array}{l}\text { Epinephelus poecilonotus (Temminck \& } \\
\text { Schlegel, 1842) }\end{array}$ & RFA & & LC & 4.0 & $\mathrm{r} 1$ \\
\hline Epinephelus retouti Bleeker, 1868 & RFA & & LC & 4.0 & $\mathrm{r} 1$ \\
\hline Epinephelus spilotoceps Schultz, 1953 & RFA & & LC & 3.7 & $\mathrm{r} 1, \mathrm{r} 11$ \\
\hline Epinephelus summana (Forsskål, 1775) & RFA & & LC & 3.8 & $\mathrm{r} 1$ \\
\hline Epinephelus tauvina (Forsskål, 1775) & RFA; OD & $\begin{array}{l}\text { Reports of ciguatera } \\
\text { poisoning }\end{array}$ & & 4.1 & $\mathrm{r} 1$ \\
\hline
\end{tabular}




\begin{tabular}{|c|c|c|c|c|c|}
\hline $\begin{array}{l}\text { Subclass, Order, Family, Genus, and } \\
\text { species }\end{array}$ & $\begin{array}{l}\text { Habitat } \\
\text { types }\end{array}$ & Threat to humans & $\begin{array}{l}\text { IUCN } \\
\text { status }\end{array}$ & $\begin{array}{l}\text { Trophic } \\
\text { level }\end{array}$ & $\begin{array}{l}\text { Other } \\
\text { remarks }\end{array}$ \\
\hline \multicolumn{6}{|l|}{ Genus Gracila } \\
\hline $\begin{array}{l}\text { Gracila albomarginata (Fowler \& Bean, } \\
\text { 1930) }\end{array}$ & RFA & & LC & 4.2 & $\mathrm{r} 1$ \\
\hline \multicolumn{6}{|l|}{ Genus Grammistes } \\
\hline Grammistes sexlineatus (Thunberg, 1792) & RFA & $\begin{array}{l}\text { Reports of ciguatera } \\
\text { poisoning }\end{array}$ & LC & 4.0 & $r 1, r 11$ \\
\hline \multicolumn{6}{|l|}{ Genus Plectropomus } \\
\hline Plectropomus areolatus (Rüppell, 1830) & RFA & $\begin{array}{l}\text { Reports of ciguatera } \\
\text { poisoning }\end{array}$ & VU & 4.5 & r1 \\
\hline Plectropomus laevis (Lacepède, 1801) & RFA & $\begin{array}{l}\text { Reports of ciguatera } \\
\text { poisoning }\end{array}$ & LC & 4.1 & r1 \\
\hline Plectropomus leopardus (Lacepède, 1802) & RFA & $\begin{array}{l}\text { Reports of ciguatera } \\
\text { poisoning }\end{array}$ & LC & 4.4 & $\mathrm{r} 1$ \\
\hline $\begin{array}{l}\text { Plectropomus oligacanthus (Bleeker, } \\
\text { 1855) }\end{array}$ & RFA & $\begin{array}{l}\text { Reports of ciguatera } \\
\text { poisoning }\end{array}$ & LC & 4.0 & $\mathrm{r} 1, \mathrm{r} 7$ \\
\hline \multicolumn{6}{|l|}{ Genus Pogonoperca } \\
\hline Pogonoperca ocellata Günther, 1859 & RFA & & LC & 4.0 & $\mathrm{r} 1$ \\
\hline \multicolumn{6}{|l|}{ Genus Pseudanthias } \\
\hline Pseudanthias cichlops (Bleeker, 1853) & RFA & & LC & 3.4 & r5 \\
\hline $\begin{array}{l}\text { Pseudanthias pascalus (Jordan \& Tanaka, } \\
\text { 1927) }\end{array}$ & RFA & & LC & 3.3 & $\mathrm{r} 6, \mathrm{r} 11$ \\
\hline $\begin{array}{l}\text { Pseudanthias tuka (Herre \& Montalban, } \\
\text { 1927) }\end{array}$ & RFA & & LC & 3.6 & $\mathrm{r} 1$ \\
\hline \multicolumn{6}{|l|}{ Genus Saloptia } \\
\hline Saloptia powelli Smith, 1964 & RFA & & LC & 3.9 & $\mathrm{r} 1$ \\
\hline \multicolumn{6}{|l|}{ Genus Variola } \\
\hline Variola louti (Forsskål, 1775) & RFA & $\begin{array}{l}\text { Reports of ciguatera } \\
\text { poisoning }\end{array}$ & LC & 4.3 & $\mathrm{r} 1$ \\
\hline \multicolumn{6}{|l|}{ Family Pseudochromidae } \\
\hline \multicolumn{6}{|l|}{ Genus Labracinus } \\
\hline $\begin{array}{l}\text { Labracinus cyclophthalmus (Müller \& } \\
\text { Troschel, 1849) }\end{array}$ & RFA & & & 3.9 & $\mathrm{r} 1$ \\
\hline Genus Pseudochromis & & & & & \\
\hline
\end{tabular}




\begin{tabular}{|c|c|c|c|c|c|}
\hline $\begin{array}{l}\text { Subclass, Order, Family, Genus, and } \\
\text { species }\end{array}$ & $\begin{array}{l}\text { Habitat } \\
\text { types }\end{array}$ & Threat to humans & $\begin{array}{l}\text { IUCN } \\
\text { status }\end{array}$ & $\begin{array}{l}\text { Trophic } \\
\text { level }\end{array}$ & $\begin{array}{l}\text { Other } \\
\text { remarks }\end{array}$ \\
\hline $\begin{array}{l}\text { Pseudochromis fuscus Müller \& Troschel, } \\
1849\end{array}$ & RFA & & LC & 3.5 & $\mathrm{r} 1$ \\
\hline \multicolumn{6}{|l|}{ Family Plesiopida } \\
\hline \multicolumn{6}{|l|}{ Genus Plesiops } \\
\hline Plesiops coeruleolineatus Rüppell, 1835 & RFA & & & 3.6 & $\mathrm{r} 1$ \\
\hline \multicolumn{6}{|l|}{ Family Priacanthidae } \\
\hline \multicolumn{6}{|l|}{ Genus Heteropriacanthus } \\
\hline $\begin{array}{l}\text { Heteropriacanthus cruentatus (Lacepède, } \\
\text { 1801) }\end{array}$ & RFA & $\begin{array}{l}\text { Reports of ciguatera } \\
\text { poisoning }\end{array}$ & LC & 3.6 & r1 \\
\hline \multicolumn{6}{|l|}{ Genus Priacanthus } \\
\hline Priacanthus hamrur (Forsskål, 1775) & RFA & & LC & 3.6 & $\mathrm{r} 1, \mathrm{r} 7$ \\
\hline Priacanthus macracanthus Cuvier, 1829 & RFA; OD & & LC & 4.1 & r6 \\
\hline \multicolumn{6}{|l|}{ Family Apogonidae } \\
\hline \multicolumn{6}{|l|}{ Genus Cheilodipterus } \\
\hline $\begin{array}{l}\text { Cheilodipterus quinquelineatus Cuvier, } \\
1828\end{array}$ & RFA & & & 3.9 & $r 1, r 8, r 11$ \\
\hline \multicolumn{6}{|l|}{ Genus Fowleria } \\
\hline Fowleria aurita (Valenciennes, 1831) & RFA & & & 3.5 & r1 \\
\hline \multicolumn{6}{|l|}{ Genus Nectamia } \\
\hline Nectamia bandanensis (Bleeker, 1854) & RFA & & & 3.3 & $\mathrm{r} 1$ \\
\hline \multicolumn{6}{|l|}{ Genus Ostorhinchus } \\
\hline $\begin{array}{l}\text { Ostorhinchus nigrofasciatus (Lachner, } \\
\text { 1953) }\end{array}$ & RFA & & & 3.6 & $\mathrm{r} 8, \mathrm{r} 11$ \\
\hline $\begin{array}{l}\text { Ostorhinchus novemfasciatus (Cuvier, } \\
\text { 1828) }\end{array}$ & RFA & & & 4.0 & $\mathrm{r} 1, \mathrm{r} 11$ \\
\hline Ostorhinchus cookii (Macleay, 1881) & RFA & & & 4.0 & $\mathrm{r} 6, \mathrm{r} 11$ \\
\hline \multicolumn{6}{|l|}{ Genus Pristicon } \\
\hline Pristicon trimaculatus (Cuvier, 1828) & RFA & & & 3.5 & r1 \\
\hline \multicolumn{6}{|l|}{ Genus Sphaeramia } \\
\hline Sphaeramia orbicularis (Cuvier, 1828) & RFA & & & 3.6 & $\mathrm{r} 1$ \\
\hline Family Malacanthidae & & & & & \\
\hline
\end{tabular}




\begin{tabular}{|c|c|c|c|c|c|}
\hline $\begin{array}{l}\text { Subclass, Order, Family, Genus, and } \\
\text { species }\end{array}$ & $\begin{array}{l}\text { Habitat } \\
\text { types }\end{array}$ & Threat to humans & $\begin{array}{l}\text { IUCN } \\
\text { status }\end{array}$ & $\begin{array}{l}\text { Trophic } \\
\text { level }\end{array}$ & $\begin{array}{l}\text { Other } \\
\text { remarks }\end{array}$ \\
\hline \multicolumn{6}{|l|}{ Genus Malacanthus } \\
\hline Malacanthus brevirostris Guichenot, 1848 & RFA & & & 3.5 & $\mathrm{r} 1$ \\
\hline Malacanthus latovittatus (Lacepède, 1801) & RFA & & & 3.5 & $\mathrm{r} 1$ \\
\hline \multicolumn{6}{|l|}{ Family Microdesmidae } \\
\hline \multicolumn{6}{|l|}{ Genus Ptereleotris } \\
\hline Ptereleotris microlepis (Bleeker, 1856) & RFA & & & 3.4 & r1 \\
\hline \multicolumn{6}{|l|}{ Family Coryphaenidae } \\
\hline \multicolumn{6}{|l|}{ Genus Coryphaena } \\
\hline Coryphaena hippurus Linnaeus, 1758 & PN; OD & $\begin{array}{l}\text { Reports of ciguatera } \\
\text { poisoning }\end{array}$ & LC & 4.4 & r1 \\
\hline \multicolumn{6}{|l|}{ Family Echeneidae } \\
\hline \multicolumn{6}{|l|}{ Genus Echeneis } \\
\hline Echeneis naucrates Linnaeus, 1758 & RFA & & LC & 3.7 & r1 \\
\hline \multicolumn{6}{|l|}{ Genus Remora } \\
\hline $\begin{array}{l}\text { Remora albescens (Temminck \& Schlegel, } \\
\text { 1850) }\end{array}$ & PEL; OD & & LC & 3.4 & $\mathrm{r} 1$ \\
\hline Remora brachyptera (Lowe, 1839) & PEL; OD & & LC & 3.5 & $\mathrm{r} 1$ \\
\hline Remora remora (Linnaeus, 1758) & RFA & & LC & 3.5 & $\mathrm{r} 1$ \\
\hline \multicolumn{6}{|l|}{ Genus Rhombochirus } \\
\hline Remora osteochir (Cuvier, 1829) & RFA & & LC & 3.5 & $\mathrm{r} 1$ \\
\hline \multicolumn{6}{|l|}{ Family Carangidae } \\
\hline \multicolumn{6}{|l|}{ Genus Carangoides } \\
\hline Carangoides ferdau (Forsskål, 1775) & RFA & $\begin{array}{l}\text { Reports of ciguatera } \\
\text { poisoning }\end{array}$ & LC & 4.3 & $\mathrm{r} 1$ \\
\hline \multicolumn{6}{|l|}{ Genus Caranx } \\
\hline Caranx ignobilis (Forsskål, 1775) & RFA & $\begin{array}{l}\text { Reports of ciguatera } \\
\text { poisoning }\end{array}$ & LC & 4.2 & $\mathrm{r} 1, \mathrm{r} 7$ \\
\hline Caranx melampygus Cuvier, 1833 & RFA & $\begin{array}{l}\text { Reports of ciguatera } \\
\text { poisoning }\end{array}$ & LC & 4.5 & $\mathrm{r} 1, \mathrm{r} 11$ \\
\hline $\begin{array}{l}\text { Caranx sexfasciatus Quoy \& Gaimard, } \\
1825\end{array}$ & RFA & & LC & 4.5 & $\mathrm{r} 1$ \\
\hline
\end{tabular}




\begin{tabular}{|c|c|c|c|c|c|}
\hline $\begin{array}{l}\text { Subclass, Order, Family, Genus, and } \\
\text { species }\end{array}$ & $\begin{array}{l}\text { Habitat } \\
\text { types }\end{array}$ & Threat to humans & $\begin{array}{l}\text { IUCN } \\
\text { status }\end{array}$ & $\begin{array}{l}\text { Trophic } \\
\text { level }\end{array}$ & $\begin{array}{l}\text { Other } \\
\text { remarks }\end{array}$ \\
\hline \multicolumn{6}{|l|}{ Genus Decapterus } \\
\hline Decapterus macrosoma Bleeker, 1851 & RFA & & LC & 3.4 & $\mathrm{r} 1, \mathrm{r} 7$ \\
\hline \multicolumn{6}{|l|}{ Genus Elagatis } \\
\hline $\begin{array}{l}\text { Elagatis bipinnulata (Quoy \& Gaimard, } \\
\text { 1825) }\end{array}$ & RFA & $\begin{array}{l}\text { Reports of ciguatera } \\
\text { poisoning }\end{array}$ & LC & 4.3 & $\mathrm{r} 1$ \\
\hline \multicolumn{6}{|l|}{ Genus Naucrates } \\
\hline Naucrates ductor (Linnaeus, 1758) & RFA & & LC & 3.4 & $\mathrm{r} 1$ \\
\hline \multicolumn{6}{|l|}{ Genus Scomberoides } \\
\hline Scomberoides lysan (Forsskål, 1775) & RFA & Other & LC & 4.0 & $\mathrm{r} 1$ \\
\hline \multicolumn{6}{|l|}{ Genus Selar } \\
\hline Selar crumenophthalmus (Bloch, 1793) & RFA & $\begin{array}{l}\text { Reports of ciguatera } \\
\text { poisoning }\end{array}$ & LC & 3.8 & $\mathrm{r} 1, \mathrm{r} 7$ \\
\hline \multicolumn{6}{|l|}{ Genus Selaroides } \\
\hline Selaroides leptolepis (Cuvier, 1833) & $\begin{array}{l}\text { RFA; } \\
\text { AMP }\end{array}$ & & LC & 3.8 & $\mathrm{r} 1$ \\
\hline \multicolumn{6}{|l|}{ Genus Seriola } \\
\hline Seriola dumerili (Risso, 1810) & RFA; OD & $\begin{array}{l}\text { Reports of ciguatera } \\
\text { poisoning }\end{array}$ & LC & 4.5 & $\mathrm{r} 1$ \\
\hline Seriola lalandi Valenciennes, 1833 & BEP & & LC & 4.2 & $\mathrm{r} 1$ \\
\hline $\begin{array}{l}\text { Seriola quinqueradiata Temminck \& } \\
\text { Schlegel, } 1845\end{array}$ & DEM; OD & & LC & 4.0 & $\mathrm{r} 1$ \\
\hline \multicolumn{6}{|l|}{ Genus Trachinotus } \\
\hline Trachinotus baillonii (Lacepède, 1801) & RFA & & LC & 3.6 & $\mathrm{r} 1$ \\
\hline \multicolumn{6}{|l|}{ Genus Alectis } \\
\hline Alectis indica (Rüppell, 1830) & RFA & & LC & 4.1 & $\mathrm{r} 1$ \\
\hline \multicolumn{6}{|l|}{ Genus Caranx } \\
\hline Caranx lugubris Poey, 1860 & BEP; OD & $\begin{array}{l}\text { Reports of ciguatera } \\
\text { poisoning }\end{array}$ & LC & 4.5 & $\mathrm{r} 1$ \\
\hline \multicolumn{6}{|l|}{ Family Bramidae } \\
\hline \multicolumn{6}{|l|}{ Genus Brama } \\
\hline Brama japonica Hilgendorf, 1878 & PEL; OD & & & 4.4 & $\mathrm{r} 1$ \\
\hline
\end{tabular}




\begin{tabular}{|c|c|c|c|c|c|}
\hline $\begin{array}{l}\text { Subclass, Order, Family, Genus, and } \\
\text { species }\end{array}$ & $\begin{array}{l}\text { Habitat } \\
\text { types }\end{array}$ & Threat to humans & $\begin{array}{l}\text { IUCN } \\
\text { status }\end{array}$ & $\begin{array}{l}\text { Trophic } \\
\text { level }\end{array}$ & $\begin{array}{l}\text { Other } \\
\text { remarks }\end{array}$ \\
\hline \multicolumn{6}{|l|}{ Family Lutjanidae } \\
\hline \multicolumn{6}{|l|}{ Genus Aphareus } \\
\hline Aphareus furca (Lacepède, 1801) & RFA & $\begin{array}{l}\text { Reports of ciguatera } \\
\text { poisoning }\end{array}$ & LC & 4.1 & $\mathrm{r} 1$ \\
\hline Aphareus rutilans Cuvier, 1830 & RFA & & LC & 4.1 & r1 \\
\hline \multicolumn{6}{|l|}{ Genus Aprion } \\
\hline Aprion virescens Valenciennes, 1830 & RFA & $\begin{array}{l}\text { Reports of ciguatera } \\
\text { poisoning }\end{array}$ & LC & 4.3 & $r 1, r 6, r 11$ \\
\hline \multicolumn{6}{|l|}{ Genus Etelis } \\
\hline Etelis carbunculus Cuvier, 1828 & BEP & & LC & 4.5 & r1 \\
\hline \multicolumn{6}{|l|}{ Genus Lutjanus } \\
\hline $\begin{array}{l}\text { Lutjanus argentimaculatus (Forsskål, } \\
\text { 1775) }\end{array}$ & RFA; OD & $\begin{array}{l}\text { Reports of ciguatera } \\
\text { poisoning }\end{array}$ & LC & 3.6 & $\mathrm{r} 1$ \\
\hline Lutjanus bohar (Forsskål, 1775) & RFA & $\begin{array}{l}\text { Reports of ciguatera } \\
\text { poisoning }\end{array}$ & LC & 4.3 & $\mathrm{r} 1$ \\
\hline Lutjanus erythropterus Bloch, 1790 & RFA & & LC & 4.5 & $\mathrm{r} 1$ \\
\hline Lutjanus fulviflamma (Forsskål, 1775) & RFA & $\begin{array}{l}\text { Reports of ciguatera } \\
\text { poisoning }\end{array}$ & LC & 3.8 & r1 \\
\hline Lutjanus fulvus (Forster, 1801) & RFA & $\begin{array}{l}\text { Reports of ciguatera } \\
\text { poisoning }\end{array}$ & LC & 3.6 & $\mathrm{r} 1, \mathrm{r} 11$ \\
\hline Lutjanus gibbus (Forsskål, 1775) & RFA & $\begin{array}{l}\text { Reports of ciguatera } \\
\text { poisoning }\end{array}$ & LC & 4.1 & $\mathrm{r} 1, \mathrm{r} 11$ \\
\hline Lutjanus kasmira (Forsskål, 1775) & RFA & & LC & 3.9 & $\mathrm{r} 1, \mathrm{r} 7$ \\
\hline Lutjanus monostigma (Cuvier, 1828) & RFA & $\begin{array}{l}\text { Reports of ciguatera } \\
\text { poisoning }\end{array}$ & LC & 4.3 & $\mathrm{r} 1$ \\
\hline Lutjanus quinquelineatus (Bloch, 1790) & RFA & & LC & 3.7 & $\mathrm{r} 1$ \\
\hline Lutjanus russellii (Bleeker, 1849) & RFA & & LC & 4.1 & $\mathrm{r} 1$ \\
\hline Lutjanus sebae (Cuvier, 1816) & RFA & $\begin{array}{l}\text { Reports of ciguatera } \\
\text { poisoning }\end{array}$ & LC & 4.1 & $\mathrm{r} 1$ \\
\hline Lutjanus stellatus Akazaki, 1983 & RFA & & & 4.0 & $\mathrm{r} 1$ \\
\hline \multicolumn{6}{|l|}{ Genus Macolor } \\
\hline Macolor niger (Forsskål, 1775) & RFA & & LC & 4.0 & $\mathrm{r} 1$ \\
\hline
\end{tabular}




\begin{tabular}{|c|c|c|c|c|c|}
\hline $\begin{array}{l}\text { Subclass, Order, Family, Genus, and } \\
\text { species }\end{array}$ & $\begin{array}{l}\text { Habitat } \\
\text { types }\end{array}$ & Threat to humans & $\begin{array}{l}\text { IUCN } \\
\text { status }\end{array}$ & $\begin{array}{l}\text { Trophic } \\
\text { level }\end{array}$ & $\begin{array}{l}\text { Other } \\
\text { remarks }\end{array}$ \\
\hline \multicolumn{6}{|l|}{ Genus Paracaesio } \\
\hline $\begin{array}{l}\text { Paracaesio sordida Abe \& Shinohara, } \\
1962\end{array}$ & RFA & & LC & 2.8 & $\mathrm{r} 1$ \\
\hline Paracaesio xanthura (Bleeker, 1869) & RFA & & LC & 3.4 & r1 \\
\hline \multicolumn{6}{|l|}{ Genus Pristipomoides } \\
\hline $\begin{array}{l}\text { Pristipomoides argyrogrammicus } \\
\text { (Valenciennes, 1832) }\end{array}$ & RFA & & LC & 4.2 & $\mathrm{r} 1$ \\
\hline $\begin{array}{l}\text { Pristipomoides auricilla (Jordan, } \\
\text { Evermann \& Tanaka, 1927) }\end{array}$ & BEP & & LC & 3.9 & $\mathrm{r} 1$ \\
\hline $\begin{array}{l}\text { Pristipomoides filamentosus } \\
\text { (Valenciennes, 1830) }\end{array}$ & BEP & & LC & 4.2 & $\mathrm{r} 1$ \\
\hline Pristipomoides flavipinnis Shinohara, 1963 & RFA & & LC & 3.6 & $\mathrm{r} 1$ \\
\hline Pristipomoides multidens (Day, 1871) & DEM & & LC & 3.8 & $\mathrm{r} 1, \mathrm{r} 7$ \\
\hline Pristipomoides sieboldii (Bleeker, 1855) & BEP & & LC & 3.7 & r5 \\
\hline \multicolumn{6}{|l|}{ Genus Symphorichthys } \\
\hline Symphorichthys spilurus (Günther, 1874) & RFA & & LC & 3.8 & $\mathrm{r} 1$ \\
\hline \multicolumn{6}{|l|}{ Family Caesionidae } \\
\hline \multicolumn{6}{|l|}{ Genus Caesio } \\
\hline Caesio caerulaurea Lacepède, 1801 & RFA & & LC & 3.4 & $\mathrm{r} 1, \mathrm{r} 11$ \\
\hline Caesio lunaris Cuvier, 1830 & RFA & & LC & 3.4 & $\mathrm{r} 1, \mathrm{r} 7$ \\
\hline Caesio teres Seale, 1906 & RFA & & LC & 3.4 & r6 \\
\hline Caesio xanthonota Bleeker, 1853 & RFA & & LC & 3.4 & $\mathrm{r} 1$ \\
\hline \multicolumn{6}{|l|}{ Genus Pterocaesio } \\
\hline Pterocaesio digramma (Bleeker, 1864) & RFA & & LC & 3.4 & $r 1, r 6, r 7$ \\
\hline Pterocaesio tile (Cuvier, 1830) & RFA & & LC & 3.3 & $r 1, r 6, r 11$ \\
\hline *Pterocaesio trilineata Carpenter, 1987 & RFA & & LC & 3.4 & $r 10, r 11$ \\
\hline \multicolumn{6}{|l|}{ Family Gerridae } \\
\hline \multicolumn{6}{|l|}{ Genus Gerres } \\
\hline Gerres filamentosus Cuvier, 1829 & $\begin{array}{l}\text { DEM; } \\
\text { AMP }\end{array}$ & & LC & 3.3 & r1 \\
\hline Gerres longirostris (Lacepède, 1801) & RFA & & LC & 3.5 & $\mathrm{r} 1$ \\
\hline
\end{tabular}




\begin{tabular}{|c|c|c|c|c|c|}
\hline $\begin{array}{l}\text { Subclass, Order, Family, Genus, and } \\
\text { species }\end{array}$ & $\begin{array}{l}\text { Habitat } \\
\text { types }\end{array}$ & Threat to humans & $\begin{array}{l}\text { IUCN } \\
\text { status }\end{array}$ & $\begin{array}{l}\text { Trophic } \\
\text { level }\end{array}$ & $\begin{array}{l}\text { Other } \\
\text { remarks }\end{array}$ \\
\hline Gerres oblongus Cuvier, 1830 & RFA & & LC & 3.5 & r1 \\
\hline Gerres oyena (Forsskål, 1775) & RFA & & LC & 2.7 & r1 \\
\hline \multicolumn{6}{|l|}{ Family Callionymidae } \\
\hline \multicolumn{6}{|l|}{ Genus Neosynchiropus } \\
\hline Neosynchiropus ocellatus (Pallas, 1770) & RFA & & & 3.2 & r1 \\
\hline \multicolumn{6}{|l|}{ Family Haemulidae } \\
\hline \multicolumn{6}{|l|}{ Genus Diagramma } \\
\hline Diagramma pictum (Thunberg, 1792) & RFA & $\begin{array}{l}\text { Reports of ciguatera } \\
\text { poisoning }\end{array}$ & & 3.7 & $\mathrm{r} 1$ \\
\hline \multicolumn{6}{|l|}{ Genus Plectorhinchus } \\
\hline $\begin{array}{l}\text { Plectorhinchus chaetodonoides Lacepède, } \\
1801\end{array}$ & RFA & & & 3.8 & $r 1, r 8, r 11$ \\
\hline $\begin{array}{l}\text { Plectorhinchus diagrammus (Linnaeus, } \\
1758 \text { ) }\end{array}$ & RFA & & & 4.0 & $\mathrm{r} 1$ \\
\hline $\begin{array}{l}\text { Plectorhinchus flavomaculatus (Cuvier, } \\
1830 \text { ) }\end{array}$ & RFA & & & 4.0 & $\mathrm{r} 1$ \\
\hline Plectorhinchus lineatus (Linnaeus, 1758) & RFA & & & 3.9 & $\mathrm{r} 1$ \\
\hline Plectorhinchus picus (Cuvier, 1828) & RFA & & & 3.9 & $\mathrm{r} 1$ \\
\hline Plectorhinchus vittatus (Linnaeus, 1758) & RFA & & LC & 3.9 & $\mathrm{r} 1, \mathrm{r} 11$ \\
\hline \multicolumn{6}{|l|}{ Family Nemipteridae } \\
\hline \multicolumn{6}{|l|}{ Genus Pentapodus } \\
\hline Pentapodus caninus (Cuvier, 1830) & RFA & & LC & 3.6 & $\mathrm{r} 1, \mathrm{r} 11$ \\
\hline Pentapodus nagasakiensis (Tanaka, 1915) & RFA & & LC & 3.4 & $\mathrm{r} 1$ \\
\hline \multicolumn{6}{|l|}{ Genus Scolopsis } \\
\hline Scolopsis aurata (Park, 1797) & RFA & & LC & 3.6 & $\mathrm{r} 1$ \\
\hline Scolopsis bilineata (Bloch, 1793) & RFA & & LC & 3.6 & $r 1, r 11$ \\
\hline Scolopsis lineata Quoy \& Gaimard, 1824 & RFA & & LC & 3.8 & $r 1, r 8, r 11$ \\
\hline Scolopsis monogramma (Cuvier, 1830) & RFA & & LC & 3.5 & r6 \\
\hline Scolopsis taenioptera (Cuvier, 1830) & DEM & & LC & 3.9 & $\mathrm{r} 1$ \\
\hline \multicolumn{6}{|l|}{ Family Lethrinidae } \\
\hline Genus Gnathodentex & & & & & \\
\hline
\end{tabular}




\begin{tabular}{|c|c|c|c|c|c|}
\hline $\begin{array}{l}\text { Subclass, Order, Family, Genus, and } \\
\text { species }\end{array}$ & $\begin{array}{l}\text { Habitat } \\
\text { types }\end{array}$ & Threat to humans & $\begin{array}{l}\text { IUCN } \\
\text { status }\end{array}$ & $\begin{array}{l}\text { Trophic } \\
\text { level }\end{array}$ & $\begin{array}{l}\text { Other } \\
\text { remarks }\end{array}$ \\
\hline $\begin{array}{l}\text { Gnathodentex aureolineatus (Lacepède, } \\
\text { 1802) }\end{array}$ & RFA & $\begin{array}{l}\text { Reports of ciguatera } \\
\text { poisoning }\end{array}$ & LC & 3.7 & $\mathrm{r} 1, \mathrm{r} 6, \mathrm{r} 7, \mathrm{r} 8$ \\
\hline \multicolumn{6}{|l|}{ Genus Gymnocranius } \\
\hline $\begin{array}{l}\text { Gymnocranius griseus (Temminck \& } \\
\text { Schlegel, 1843) }\end{array}$ & RFA & & LC & 3.2 & $\mathrm{r} 1, \mathrm{r} 7$ \\
\hline \multicolumn{6}{|l|}{ Genus Lethrinus } \\
\hline $\begin{array}{l}\text { Lethrinus haematopterus Temminck \& } \\
\text { Schlegel, } 1844\end{array}$ & RFA & & & 3.7 & $\mathrm{r} 1$ \\
\hline Lethrinus lentjan (Lacepède, 1802) & RFA & & LC & 3.9 & $\mathrm{r} 1, \mathrm{r} 7$ \\
\hline Lethrinus miniatus (Forster, 1801) & RFA & $\begin{array}{l}\text { Reports of ciguatera } \\
\text { poisoning }\end{array}$ & LC & 4.2 & r1 \\
\hline Lethrinus nebulosus (Forsskål, 1775) & RFA & $\begin{array}{l}\text { Reports of ciguatera } \\
\text { poisoning }\end{array}$ & LC & 3.8 & $r 1, r 6$ \\
\hline Lethrinus ornatus Valenciennes, 1830 & RFA & & LC & 3.4 & $\mathrm{r} 1$ \\
\hline Lethrinus rubrioperculatus Sato, 1978 & RFA & & LC & 3.8 & $\mathrm{r} 1, \mathrm{r} 11$ \\
\hline Lethrinus variegatus Valenciennes, 1830 & RFA & & LC & 3.8 & $\mathrm{r} 1, \mathrm{r} 7$ \\
\hline Lethrinus xanthochilus Klunzinger, 1870 & RFA & & LC & 3.8 & $\mathrm{r} 1$ \\
\hline \multicolumn{6}{|l|}{ Genus Monotaxis } \\
\hline Monotaxis grandoculis (Forsskål, 1775) & RFA & $\begin{array}{l}\text { Reports of ciguatera } \\
\text { poisoning }\end{array}$ & LC & 3.4 & $\mathrm{r} 1, \mathrm{r} 8, \mathrm{r} 11$ \\
\hline \multicolumn{6}{|l|}{ Genus Pentapodus } \\
\hline Pentapodus setosus (Valenciennes, 1830) & RFA & & & 3.5 & $\mathrm{r} 1$ \\
\hline \multicolumn{6}{|l|}{ Family Sparidae } \\
\hline \multicolumn{6}{|l|}{ Genus Dentex } \\
\hline $\begin{array}{l}\text { Dentex tumifrons (Temminck \& Schlegel, } \\
1843 \text { ) }\end{array}$ & DEM & & LC & 3.8 & $\mathrm{r} 1$ \\
\hline \multicolumn{6}{|l|}{ Family Mullidae } \\
\hline \multicolumn{6}{|l|}{ Genus Mulloidichthys } \\
\hline $\begin{array}{l}\text { Mulloidichthys flavolineatus (Lacepède, } \\
\text { 1801) }\end{array}$ & RFA & & LC & 3.8 & $\mathrm{r} 1$ \\
\hline $\begin{array}{l}\text { Mulloidichthys vanicolensis (Valenciennes, } \\
\text { 1831) }\end{array}$ & RFA & & LC & 3.6 & $\mathrm{r} 1, \mathrm{r} 6$ \\
\hline
\end{tabular}




\begin{tabular}{|c|c|c|c|c|c|}
\hline $\begin{array}{l}\text { Subclass, Order, Family, Genus, and } \\
\text { species }\end{array}$ & $\begin{array}{l}\text { Habitat } \\
\text { types }\end{array}$ & Threat to humans & $\begin{array}{l}\text { IUCN } \\
\text { status }\end{array}$ & $\begin{array}{l}\text { Trophic } \\
\text { level }\end{array}$ & $\begin{array}{l}\text { Other } \\
\text { remarks }\end{array}$ \\
\hline \multicolumn{6}{|l|}{ Genus Parupeneus } \\
\hline Parupeneus barberinus (Lacepède, 1801) & RFA & & LC & 3.4 & $r 1, r 6, r 7, r 8, r 11$ \\
\hline Parupeneus ciliatus (Lacepède, 1802) & RFA & & LC & 3.5 & $\mathrm{r} 1, \mathrm{r} 6$ \\
\hline $\begin{array}{l}\text { Parupeneus crassilabris (Valenciennes, } \\
\text { 1831) }\end{array}$ & RFA & & LC & 3.6 & r6 \\
\hline $\begin{array}{l}\text { Parupeneus cyclostomus (Lacepède, } \\
\text { 1801) }\end{array}$ & RFA & $\begin{array}{l}\text { Reports of ciguatera } \\
\text { poisoning }\end{array}$ & LC & 4.2 & $r 1, r 6, r 8, r 11$ \\
\hline $\begin{array}{l}\text { Parupeneus forsskali (Fourmanoir \& } \\
\text { Guézé, 1976) }\end{array}$ & RFA & & & 3.5 & $r 4, r 6$ \\
\hline $\begin{array}{l}\text { Parupeneus multifasciatus (Quoy \& } \\
\text { Gaimard, 1825) }\end{array}$ & RFA & & LC & 3.5 & r6,r8,r11 \\
\hline Parupeneus pleurostigma (Bennett, 1831) & RFA & & LC & 3.4 & $r 1, r 6$ \\
\hline Parupeneus trifasciatus (Lacepède, 1801) & RFA & & & 3.5 & $\mathrm{r} 1, \mathrm{r} 11$ \\
\hline $\begin{array}{l}\text { Parupeneus heptacanthus (Lacepède, } \\
\text { 1802) }\end{array}$ & RFA & & LC & 3.4 & r7 \\
\hline \multicolumn{6}{|l|}{ Genus Upeneus } \\
\hline $\begin{array}{l}\text { Upeneus subvittatus (Temminck \& } \\
\text { Schlegel, 1843) }\end{array}$ & DEM & & & 4.2 & r1 \\
\hline \multicolumn{6}{|l|}{ Family Pempheridae } \\
\hline \multicolumn{6}{|l|}{ Genus Pempheris } \\
\hline Pempheris oualensis Cuvier, 1831 & RFA & & & 3.6 & r2,r6 \\
\hline \multicolumn{6}{|l|}{ Family Kyphosidae } \\
\hline \multicolumn{6}{|l|}{ Genus Kyphosus } \\
\hline Kyphosus cinerascens (Forsskål, 1775) & RFA & Poisonous to eat & LC & 2.9 & $\mathrm{r} 1$ \\
\hline $\begin{array}{l}\text { Kyphosus vaigiensis (Quoy \& Gaimard, } \\
\text { 1825) }\end{array}$ & RFA; OD & & LC & 2.0 & $\mathrm{r} 1$ \\
\hline \multicolumn{6}{|l|}{ Family Chaetodontidae } \\
\hline \multicolumn{6}{|l|}{ Genus Chaetodon } \\
\hline Chaetodon adiergastos Seale, 1910 & RFA & & LC & 3.5 & $\mathrm{r} 1$ \\
\hline Chaetodon auriga Forsskål, 1775 & RFA & & LC & 3.7 & $\mathrm{r} 1, \mathrm{r} 8, \mathrm{r} 11$ \\
\hline Chaetodon auripes Jordan \& Snyder, 1901 & RFA & & LC & 3.5 & r6,r11 \\
\hline Chaetodon bennetti Cuvier, 1831 & RFA & & & 3.1 & $\mathrm{r} 1,9$ \\
\hline
\end{tabular}




\begin{tabular}{|c|c|c|c|c|c|}
\hline $\begin{array}{l}\text { Subclass, Order, Family, Genus, and } \\
\text { species }\end{array}$ & $\begin{array}{l}\text { Habitat } \\
\text { types }\end{array}$ & Threat to humans & $\begin{array}{l}\text { IUCN } \\
\text { status }\end{array}$ & $\begin{array}{l}\text { Trophic } \\
\text { level }\end{array}$ & $\begin{array}{l}\text { Other } \\
\text { remarks }\end{array}$ \\
\hline Chaetodon citrinellus Cuvier, 1831 & RFA & & LC & 3.5 & $\mathrm{r} 1, \mathrm{r} 11$ \\
\hline Chaetodon collare Bloch, 1787 & RFA & & LC & 3.4 & $\mathrm{r} 1$ \\
\hline Chaetodon ephippium Cuvier, 1831 & RFA & & LC & 3.0 & $\mathrm{r} 1, \mathrm{r} 8$ \\
\hline Chaetodon falcula Bloch, 1795 & RFA & & LC & 3.5 & $\mathrm{r} 1$ \\
\hline Chaetodon guttatissimus Bennett, 1833 & RFA & & LC & 3.1 & $\mathrm{r} 1$ \\
\hline Chaetodon kleinii Bloch, 1790 & RFA & & LC & 2.9 & $\mathrm{r} 1, \mathrm{r} 11$ \\
\hline Chaetodon lineolatus Cuvier, 1831 & RFA & & LC & 3.4 & r1.r11 \\
\hline Chaetodon lunula (Lacepède, 1802) & RFA & & LC & 3.7 & $r 1, r 6, r 8$ \\
\hline Chaetodon madagaskariensis Ahl, 1923 & RFA & & LC & 2.8 & $r 1, r 6, r 11$ \\
\hline $\begin{array}{l}\text { Chaetodon melannotus Bloch \& } \\
\text { Schneider, } 1801\end{array}$ & RFA & & LC & 4.4 & $r 1, r 6, r 8$ \\
\hline Chaetodon ornatissimus Cuvier, 1831 & RFA & & LC & 3.3 & $r 1, r 6, r 11$ \\
\hline Chaetodon punctatofasciatus Cuvier, 1831 & RFA & & LC & 3.4 & $\mathrm{r} 1, \mathrm{r} 6, \mathrm{r} 11$ \\
\hline $\begin{array}{l}\text { Chaetodon rafflesii Anonymous [Bennett], } \\
1830\end{array}$ & RFA & & LC & 4.3 & $\mathrm{r} 2, \mathrm{r} 6, \mathrm{r} 11$ \\
\hline Chaetodon semeion Bleeker, 1855 & RFA & & LC & 2.7 & $\mathrm{r} 1$ \\
\hline Chaetodon speculum Cuvier, 1831 & RFA & & LC & 3.6 & $\mathrm{r} 1, \mathrm{r} 11$ \\
\hline $\begin{array}{l}\text { Chaetodon trifascialis Quoy \& Gaimard, } \\
1825\end{array}$ & RFA & & NT & 3.3 & $\mathrm{r} 1, \mathrm{r} 6, \mathrm{r} 11$ \\
\hline Chaetodon trifasciatus Park, 1797 & RFA & & LC & 3.3 & $\mathrm{r} 1$ \\
\hline Chaetodon ulietensis Cuvier, 1831 & RFA & & LC & 2.7 & r6 \\
\hline Chaetodon unimaculatus Bloch, 1787 & RFA & & LC & 3.3 & r1,r6 \\
\hline Chaetodon vagabundus Linnaeus, 1758 & RFA & & LC & 2.9 & $\mathrm{r} 1, \mathrm{r} 6, \mathrm{r} 11$ \\
\hline Chaetodon wiebeli Kaup, 1863 & RFA & & LC & 2.7 & r1,r6 \\
\hline Chaetodon xanthurus Bleeker, 1857 & RFA & & LC & 2.8 & r6,r7,r8,r11 \\
\hline $\begin{array}{l}\text { Chaetodon lunulatus Quoy \& Gaimard, } \\
1825\end{array}$ & RFA & & LC & 3.3 & $\mathrm{r} 10, \mathrm{r} 11$ \\
\hline \multicolumn{6}{|l|}{ Genus Coradion } \\
\hline Coradion chrysozonus (Cuvier, 1831) & RFA & & LC & 2.8 & r1 \\
\hline Genus Forcipiger & & & & & \\
\hline
\end{tabular}




\begin{tabular}{|c|c|c|c|c|c|}
\hline $\begin{array}{l}\text { Subclass, Order, Family, Genus, and } \\
\text { species }\end{array}$ & $\begin{array}{l}\text { Habitat } \\
\text { types }\end{array}$ & Threat to humans & $\begin{array}{l}\text { IUCN } \\
\text { status }\end{array}$ & $\begin{array}{l}\text { Trophic } \\
\text { level }\end{array}$ & $\begin{array}{l}\text { Other } \\
\text { remarks }\end{array}$ \\
\hline $\begin{array}{l}\text { Forcipiger flavissimus Jordan \& McGregor, } \\
1898\end{array}$ & RFA & & LC & 3.1 & $\mathrm{r} 6, \mathrm{r} 11$ \\
\hline Forcipiger longirostris (Broussonet, 1782) & RFA & & LC & 3.5 & $r 1, r 11$ \\
\hline \multicolumn{6}{|l|}{ Genus Hemitaurichthys } \\
\hline Hemitaurichthys polylepis (Bleeker, 1857) & RFA & & LC & 3.1 & $r 6, r 11$ \\
\hline Hemitaurichthys zoster (Bennett, 1831) & RFA & & LC & 3.3 & $\mathrm{r} 1$ \\
\hline \multicolumn{6}{|l|}{ Genus Heniochus } \\
\hline Heniochus acuminatus (Linnaeus, 1758) & RFA & & LC & 3.5 & r1 \\
\hline Heniochus chrysostomus Cuvier, 1831 & RFA & & LC & 3.8 & $r 1, r 8, r 11$ \\
\hline Heniochus monoceros Cuvier, 1831 & RFA & & LC & 3.5 & $\mathrm{r} 1$ \\
\hline $\begin{array}{l}\text { Heniochus singularius Smith \& Radcliffe, } \\
1911\end{array}$ & RFA & & LC & 3.6 & $\mathrm{r} 1$ \\
\hline Heniochus varius (Cuvier, 1829) & RFA & & LC & 3.2 & $\mathrm{r} 1$ \\
\hline \multicolumn{6}{|l|}{ Genus Roa } \\
\hline $\begin{array}{l}\text { Roa modesta (Temminck \& Schlegel, } \\
\text { 1844) }\end{array}$ & RFA; OD & & LC & 3.5 & r1 \\
\hline \multicolumn{6}{|l|}{ Genus Parachaetodon } \\
\hline Parachaetodon ocellatus (Cuvier, 1831) & RFA & & LC & 2.8 & r1 \\
\hline \multicolumn{6}{|l|}{ Family Pomacanthidae } \\
\hline \multicolumn{6}{|l|}{ Genus Apolemichthys } \\
\hline Apolemichthys trimaculatus (Cuvier, 1831) & RFA & & LC & 2.6 & $\mathrm{r} 1, \mathrm{r} 11$ \\
\hline \multicolumn{6}{|l|}{ Genus Centropyge } \\
\hline Centropyge bispinosa (Günther, 1860) & RFA & & LC & 2.8 & $r 1, r 6, r 8, r 11$ \\
\hline $\begin{array}{l}\text { Centropyge heraldi Woods \& Schultz, } \\
1953\end{array}$ & RFA & & LC & 2.8 & $\mathrm{r} 1, \mathrm{r} 11$ \\
\hline Centropyge vrolikii (Bleeker, 1853) & RFA & & LC & 2.8 & $\mathrm{r} 1, \mathrm{r} 6$ \\
\hline${ }^{*}$ Centropyge tibicen (Cuvier, 1831) & RFA & & LC & 2.8 & r11 \\
\hline \multicolumn{6}{|l|}{ Genus Genicanthus } \\
\hline Genicanthus melanospilos (Bleeker, 1857) & RFA & & LC & 3.4 & r1 \\
\hline \multicolumn{6}{|l|}{ Genus Pomacanthus } \\
\hline Pomacanthus annularis (Bloch, 1787) & RFA & & LC & 2.6 & $\mathrm{r} 1$ \\
\hline
\end{tabular}




\begin{tabular}{|c|c|c|c|c|c|}
\hline $\begin{array}{l}\text { Subclass, Order, Family, Genus, and } \\
\text { species }\end{array}$ & $\begin{array}{l}\text { Habitat } \\
\text { types }\end{array}$ & Threat to humans & $\begin{array}{l}\text { IUCN } \\
\text { status }\end{array}$ & $\begin{array}{l}\text { Trophic } \\
\text { level }\end{array}$ & $\begin{array}{l}\text { Other } \\
\text { remarks }\end{array}$ \\
\hline Pomacanthus imperator (Bloch, 1787) & RFA & & LC & 2.7 & $\mathrm{r} 1, \mathrm{r} 6, \mathrm{r} 11$ \\
\hline $\begin{array}{l}\text { Pomacanthus semicirculatus (Cuvier, } \\
\text { 1831) }\end{array}$ & RFA & & LC & 2.7 & $\mathrm{r} 1$ \\
\hline Pomacanthus sexstriatus (Cuvier, 1831) & RFA & & LC & 2.6 & $\mathrm{r} 1, \mathrm{r} 6$ \\
\hline \multicolumn{6}{|l|}{ Genus Pygoplites } \\
\hline Pygoplites diacanthus (Boddaert, 1772) & RFA & & LC & 2.7 & $\mathrm{r} 1, \mathrm{r} 6, \mathrm{r} 11$ \\
\hline \multicolumn{6}{|l|}{ Family Pentacerotidae } \\
\hline \multicolumn{6}{|l|}{ Genus Histiopterus } \\
\hline $\begin{array}{l}\text { Histiopterus typus Temminck \& Schlegel, } \\
1844\end{array}$ & RFA & & & 3.5 & $\mathrm{r} 1$ \\
\hline \multicolumn{6}{|l|}{ Family Theraponidae } \\
\hline \multicolumn{6}{|l|}{ Genus Therapon } \\
\hline Terapon jarbua (Forsskål, 1775) & DEM & & LC & 3.9 & $\mathrm{r} 1$ \\
\hline Terapon theraps Cuvier, 1829 & RFA & & LC & 3.5 & r1 \\
\hline \multicolumn{6}{|l|}{ Family Kuhliidae } \\
\hline \multicolumn{6}{|l|}{ Genus Kuhlia } \\
\hline Kuhlia mugil (Forster, 1801) & RFA & & LC & 3.8 & $\mathrm{r} 1$ \\
\hline \multicolumn{6}{|l|}{ Family Cirrhitidae } \\
\hline \multicolumn{6}{|l|}{ Genus Cirrhitichthys } \\
\hline $\begin{array}{l}\text { Cirrhitichthys aureus (Temminck \& } \\
\text { Schlegel, 1842) }\end{array}$ & RFA & & & 4.0 & $\mathrm{r} 1$ \\
\hline Cirrhitichthys falco Randall, 1963 & RFA & & LC & 4.0 & $r 6, r 11$ \\
\hline Cirrhitichthys oxycephalus (Bleeker, 1855) & RFA & & LC & 4.0 & $r 9, r 10, r 11$ \\
\hline \multicolumn{6}{|l|}{ Genus Cirrhitus } \\
\hline Cirrhitus pinnulatus (Forster, 1801) & RFA & & LC & 3.7 & $r 1, r 11$ \\
\hline \multicolumn{6}{|l|}{ Genus Paracirrhites } \\
\hline Paracirrhites arcatus (Cuvier, 1829) & RFA & & LC & 3.6 & $\mathrm{r} 1, \mathrm{r} 6, \mathrm{r} 11$ \\
\hline \multicolumn{6}{|l|}{ Family Pomacentridae } \\
\hline \multicolumn{6}{|l|}{ Genus Abudefduf } \\
\hline Abudefduf septemfasciatus (Cuvier, 1830) & RFA & & LC & 3.0 & r1 \\
\hline Abudefduf sexfasciatus (Lacepède, 1801) & RFA & & LC & 2.7 & $\mathrm{r} 1, \mathrm{r} 11$ \\
\hline
\end{tabular}




\begin{tabular}{|c|c|c|c|c|c|}
\hline $\begin{array}{l}\text { Subclass, Order, Family, Genus, and } \\
\text { species }\end{array}$ & $\begin{array}{l}\text { Habitat } \\
\text { types }\end{array}$ & Threat to humans & $\begin{array}{l}\text { IUCN } \\
\text { status }\end{array}$ & $\begin{array}{l}\text { Trophic } \\
\text { level }\end{array}$ & $\begin{array}{l}\text { Other } \\
\text { remarks }\end{array}$ \\
\hline Abudefduf sordidus (Forsskål, 1775) & RFA & & LC & 2.9 & $\mathrm{r} 1, \mathrm{r} 6, \mathrm{r} 7$ \\
\hline $\begin{array}{l}\text { Abudefduf vaigiensis (Quoy \& Gaimard, } \\
\text { 1825) }\end{array}$ & RFA & $\begin{array}{l}\text { Reports of ciguatera } \\
\text { poisoning }\end{array}$ & LC & 2.6 & $r 1, r 6, r 8, r 11$ \\
\hline${ }^{*}$ Abudefduf bengalensis (Bloch, 1787) & RFA & & LC & 3.1 & $\mathrm{r} 11$ \\
\hline \multicolumn{6}{|l|}{ Genus Amblyglyphidodon } \\
\hline Amblyglyphidodon aureus (Cuvier, 1830) & RFA & & LC & 2.7 & $\mathrm{r} 1$ \\
\hline $\begin{array}{l}\text { Amblyglyphidodon leucogaster (Bleeker, } \\
\text { 1847) }\end{array}$ & RFA & & LC & 3.4 & $r 1, r 6, r 11$ \\
\hline Amblyglyphidodon curacao (Bloch, 1787) & RFA & & LC & 2.6 & $\mathrm{r} 8, \mathrm{r} 11$ \\
\hline \multicolumn{6}{|l|}{ Genus Amphiprion } \\
\hline Amphiprion akallopisos Bleeker, 1853 & RFA & & LC & 2.7 & $\mathrm{r} 1$ \\
\hline Amphiprion bicinctus Rüppell, 1830 & RFA & & LC & 2.7 & $\mathrm{r} 1$ \\
\hline Amphiprion clarkii (Bennett, 1830) & RFA & & & 2.9 & $\mathrm{r} 6, \mathrm{r} 11$ \\
\hline Amphiprion frenatus Brevoort, 1856 & RFA & & LC & 2.7 & r1,r6,r11 \\
\hline Amphiprion perideraion Bleeker, 1855 & RFA & & LC & 2.2 & $\mathrm{r} 1, \mathrm{r} 6$ \\
\hline${ }^{*}$ Amphiprion percula (Lacepède, 1802) & RFA & & LC & 2.7 & r11 \\
\hline \multicolumn{6}{|l|}{ Genus Chromis } \\
\hline Chromis chrysura (Bliss, 1883) & RFA & & & 3.0 & $\mathrm{r} 1$ \\
\hline Chromis dimidiata (Klunzinger, 1871) & RFA & & LC & 2.7 & r1 \\
\hline Chromis margaritifer Fowler, 1946 & RFA & & & 3.0 & $\mathrm{r} 6, \mathrm{r} 11$ \\
\hline $\begin{array}{l}\text { Chromis notata (Temminck \& Schlegel, } \\
1843 \text { ) }\end{array}$ & RFA & & & 3.4 & r1 \\
\hline Chromis ternatensis (Bleeker, 1856) & RFA & & & 3.4 & $\mathrm{r} 1, \mathrm{r} 6$ \\
\hline Chromis xanthura (Bleeker, 1854) & RFA & & & 3.4 & $\mathrm{r} 1, \mathrm{r} 11$ \\
\hline${ }^{*}$ Chromis fumea (Tanaka, 1917) & RFA & & LC & 3.4 & r11 \\
\hline${ }^{*}$ Chromis lepidolepis Bleeker, 1877 & RFA & & & 3.4 & r11 \\
\hline Chromis ovatiformes Fowler, 1946 & RFA & & & 3.4 & r9,r10,r11 \\
\hline${ }^{*}$ Chromis caudalis Randall, 1988 & RFA & & LC & 3.0 & r11 \\
\hline${ }^{*}$ Chromis viridis (Cuvier, 1830) & RFA & & & 2.9 & r9,r11 \\
\hline Genus Chrysiptera & & & & & \\
\hline
\end{tabular}




\begin{tabular}{|c|c|c|c|c|c|}
\hline $\begin{array}{l}\text { Subclass, Order, Family, Genus, and } \\
\text { species }\end{array}$ & $\begin{array}{l}\text { Habitat } \\
\text { types }\end{array}$ & Threat to humans & $\begin{array}{l}\text { IUCN } \\
\text { status }\end{array}$ & $\begin{array}{l}\text { Trophic } \\
\text { level }\end{array}$ & $\begin{array}{l}\text { Other } \\
\text { remarks }\end{array}$ \\
\hline $\begin{array}{l}\text { Chrysiptera biocellata (Quoy \& Gaimard, } \\
\text { 1825) }\end{array}$ & RFA & & & 2.0 & $\mathrm{r} 1, \mathrm{r} 8, \mathrm{r} 11$ \\
\hline Chrysiptera brownriggii (Bennett, 1828) & RFA & & & 2.7 & $\mathrm{r} 1$ \\
\hline $\begin{array}{l}\text { Chrysiptera cyanea (Quoy \& Gaimard, } \\
\text { 1825) }\end{array}$ & RFA & & & 2.5 & $\mathrm{r} 1, \mathrm{r} 6$ \\
\hline Chrysiptera glauca (Cuvier, 1830) & RFA & & & 2.4 & $\mathrm{r} 1$ \\
\hline $\begin{array}{l}\text { Chrysiptera chrysocephala Manica, Pilcher } \\
\text { \& Oakley, } 2002\end{array}$ & PN & & & 2.7 & r9,r11 \\
\hline${ }^{*}$ Chrysiptera unimaculata (Cuvier, 1830) & RFA & & LC & 2.1 & $\mathrm{r} 11$ \\
\hline${ }^{*}$ Chrysiptera talboti (Allen, 1975) & RFA & & & 2.8 & $\mathrm{r} 11$ \\
\hline \multicolumn{6}{|l|}{ Genus Dascyllus } \\
\hline Dascyllus aruanus (Linnaeus, 1758) & RFA & & & 3.3 & $\mathrm{r} 1, \mathrm{r} 8, \mathrm{r} 11$ \\
\hline Dascyllus marginatus (Rüppell, 1829) & RFA & & & 2.7 & $\mathrm{r} 1$ \\
\hline Dascyllus reticulatus (Richardson, 1846) & RFA & & & 3.1 & r6 \\
\hline Dascyllus trimaculatus (Rüppell, 1829) & RFA & & & 2.8 & $\mathrm{r} 1, \mathrm{r} 6, \mathrm{r} 11$ \\
\hline Dascyllus reticulatus (Richardson, 1846) & RFA & & & 3.1 & r9,r10,r11 \\
\hline \multicolumn{6}{|l|}{ Genus Dischistodus } \\
\hline Dischistodus melanotus (Bleeker, 1858) & RFA & & & 2.0 & $\mathrm{r} 1, \mathrm{r6}, \mathrm{r} 8 . \mathrm{r11}$ \\
\hline Dischistodus perspicillatus (Cuvier, 1830) & RFA & & & 2.0 & $\mathrm{r} 1, \mathrm{r} 6, \mathrm{r} 8, \mathrm{r} 11$ \\
\hline $\begin{array}{l}\text { *Dischistodus pseudochrysopoecilus } \\
\text { (Allen \& Robertson, 1974) }\end{array}$ & RFA & & & 2.0 & $\mathrm{r} 11$ \\
\hline $\begin{array}{l}\text { Dischistodus prosopotaenia (Bleeker, } \\
\text { 1852) }\end{array}$ & & & & 2.7 & $\mathrm{r} 8, \mathrm{r} 11$ \\
\hline $\begin{array}{l}\text { *Dischistodus chrysopoecilus (Schlegel \& } \\
\text { Müller, 1839) }\end{array}$ & RFA & & & & $\mathrm{r} 11$ \\
\hline \multicolumn{6}{|l|}{ Genus Hemiglyphidodon } \\
\hline $\begin{array}{l}\text { Hemiglyphidodon plagiometopon (Bleeker, } \\
\text { 1852) }\end{array}$ & RFA & & & 2.0 & $\mathrm{r} 1$ \\
\hline \multicolumn{6}{|l|}{ Genus Neoglyphidodon } \\
\hline Neoglyphidodon melas (Cuvier, 1830) & RFA & & & 3.4 & $\mathrm{r} 1, \mathrm{r} 6$ \\
\hline $\begin{array}{l}\text { Neoglyphidodon thoracotaeniatus (Fowler } \\
\text { \& Bean, 1928) }\end{array}$ & RFA & & & 2.7 & $\mathrm{r} 1, \mathrm{r} 6$ \\
\hline
\end{tabular}




\begin{tabular}{|c|c|c|c|c|c|}
\hline $\begin{array}{l}\text { Subclass, Order, Family, Genus, and } \\
\text { species }\end{array}$ & $\begin{array}{l}\text { Habitat } \\
\text { types }\end{array}$ & Threat to humans & $\begin{array}{l}\text { IUCN } \\
\text { status }\end{array}$ & $\begin{array}{l}\text { Trophic } \\
\text { level }\end{array}$ & $\begin{array}{l}\text { Other } \\
\text { remarks }\end{array}$ \\
\hline \multicolumn{6}{|l|}{ Genus Neopomacentrus } \\
\hline Neopomacentrus azysron (Bleeker, 1877) & RFA & & & 3.4 & r6 \\
\hline \multicolumn{6}{|l|}{ Genus Plectroglyphidodon } \\
\hline Plectroglyphidodon dickii (Liénard, 1839) & RFA & & & 3.7 & $\mathrm{r} 1, \mathrm{r} 6, \mathrm{r} 11$ \\
\hline $\begin{array}{l}\text { Plectroglyphidodon lacrymatus (Quoy \& } \\
\text { Gaimard, 1825) }\end{array}$ & RFA & & & 2.2 & $r 1, r 6, r 8, r 11$ \\
\hline $\begin{array}{l}\text { Plectroglyphidodon leucozonus (Bleeker, } \\
\text { 1859) }\end{array}$ & RFA & & & 2.0 & r8 \\
\hline \multicolumn{6}{|l|}{ Genus Pomacentrus } \\
\hline Pomacentrus brachialis Cuvier, 1830 & RFA & & & 2.6 & $\mathrm{r} 1$ \\
\hline $\begin{array}{l}\text { Pomacentrus coelestis Jordan \& Starks, } \\
1901\end{array}$ & RFA & & & 3.2 & r6,r8,r11 \\
\hline Pomacentrus moluccensis Bleeker, 1853 & RFA & & & 2.4 & $\mathrm{r} 1, \mathrm{r} 6, \mathrm{r} 8, \mathrm{r} 11$ \\
\hline Pomacentrus pavo (Bloch, 1787) & RFA & & & 3.0 & $\mathrm{r} 1, \mathrm{r} 11$ \\
\hline $\begin{array}{l}\text { Pomacentrus philippinus Evermann \& } \\
\text { Seale, } 1907\end{array}$ & RFA & & & 2.7 & $\mathrm{r} 1, \mathrm{r} 6, \mathrm{r} 8, \mathrm{r} 11$ \\
\hline Pomacentrus tripunctatus Cuvier, 1830 & RFA & & & 2.0 & $\mathrm{r} 1$ \\
\hline Pomacentrus vaiuli Jordan \& Seale, 1906 & RFA & & & 3.1 & r8,r11 \\
\hline Pomacentrus bankanensis Bleeker, 1854 & RFA & & & 2.7 & r8,r11 \\
\hline \multicolumn{6}{|l|}{ Genus Pomachromis } \\
\hline Pomachromis richardsoni (Snyder, 1909) & RFA & & & 3.0 & $\mathrm{r} 1$ \\
\hline \multicolumn{6}{|l|}{ Genus Stegastes } \\
\hline $\begin{array}{l}\text { Stegastes albifasciatus (Schlegel \& Müller, } \\
\text { 1839) }\end{array}$ & RFA & & & 2.0 & $\mathrm{r} 1, \mathrm{r} 8$ \\
\hline Stegastes fasciolatus (Ogilby, 1889) & RFA & & & 2.2 & $\mathrm{r} 1, \mathrm{r} 8, \mathrm{r} 11$ \\
\hline Stegastes lividus (Forster, 1801) & RFA & & & & $\mathrm{r} 1$ \\
\hline *Stegastes insularis Allen \& Emery, 1985 & RFA & & & 2.0 & $\mathrm{r} 11$ \\
\hline Stegastes lividus (Forster, 1801) & RFA & & & 2.0 & $\mathrm{r} 1$ \\
\hline Stegastes nigricans (Lacepède, 1802) & RFA & Traumatogenic & & 2.2 & $\mathrm{r} 1$ \\
\hline \multicolumn{6}{|l|}{ Family Labridae } \\
\hline Genus Anampses & & & & & \\
\hline
\end{tabular}




\begin{tabular}{|c|c|c|c|c|c|}
\hline $\begin{array}{l}\text { Subclass, Order, Family, Genus, and } \\
\text { species }\end{array}$ & $\begin{array}{l}\text { Habitat } \\
\text { types }\end{array}$ & Threat to humans & $\begin{array}{l}\text { IUCN } \\
\text { status }\end{array}$ & $\begin{array}{l}\text { Trophic } \\
\text { level }\end{array}$ & $\begin{array}{l}\text { Other } \\
\text { remarks }\end{array}$ \\
\hline $\begin{array}{l}\text { Anampses caeruleopunctatus Rüppell, } \\
1829\end{array}$ & RFA & & & 3.4 & $\mathrm{r} 1$ \\
\hline Anampses melanurus Bleeker, 1857 & RFA & & LC & 3.4 & $\mathrm{r} 1, \mathrm{r} 11$ \\
\hline $\begin{array}{l}\text { Anampses meleagrides Valenciennes, } \\
1840\end{array}$ & RFA & & LC & 3.5 & $\mathrm{r} 1$ \\
\hline Anampses twistii Bleeker, 1856 & RFA & & LC & 3.5 & $\mathrm{r} 1$ \\
\hline \multicolumn{6}{|l|}{ Genus Bodianus } \\
\hline Bodianus axillaris (Bennett, 1832) & RFA & & LC & 3.4 & $\mathrm{r} 1, \mathrm{r} 11$ \\
\hline Bodianus bilunulatus (Lacepède, 1801) & RFA & & LC & 3.4 & $\mathrm{r} 1, \mathrm{r} 8$ \\
\hline Bodianus loxozonus (Snyder, 1908) & RFA & & LC & 3.6 & r6 \\
\hline Bodianus macrourus (Lacepède, 1801) & RFA & & LC & 3.5 & $\mathrm{r} 1$ \\
\hline $\begin{array}{l}\text { Bodianus mesothorax (Bloch \& Schneider, } \\
\text { 1801) }\end{array}$ & RFA & & LC & 3.2 & r6 \\
\hline Bodianus oxycephalus (Bleeker, 1862) & RFA & & & 3.5 & $\mathrm{r} 1$ \\
\hline \multicolumn{6}{|l|}{ Genus Cheilinus } \\
\hline Cheilinus chlorourus (Bloch, 1791) & RFA & & LC & 3.5 & $\mathrm{r} 1, \mathrm{r} 6$ \\
\hline Cheilinus fasciatus (Bloch, 1791) & RFA & & LC & 3.4 & $r 1, r 6, r 8$ \\
\hline Cheilinus oxycephalus Bleeker, 1853 & RFA & & LC & 3.4 & r1 \\
\hline Cheilinus trilobatus Lacepède, 1801 & RFA & & LC & 3.9 & $\mathrm{r} 1, \mathrm{r} 11$ \\
\hline Cheilinus undulatus Rüppell, 1835 & RFA & $\begin{array}{l}\text { Reports of ciguatera } \\
\text { poisoning }\end{array}$ & EN & 4.0 & $\mathrm{r} 1, \mathrm{r} 11$ \\
\hline \multicolumn{6}{|l|}{ Genus Cheilio } \\
\hline Cheilio inermis (Forsskål, 1775) & RFA & & LC & 3.5 & $\mathrm{r} 1$ \\
\hline $\begin{array}{l}\text { Choerodon melanostigma Fowler \& Bean, } \\
1928\end{array}$ & RFA & & LC & 3.5 & $\mathrm{r} 1$ \\
\hline $\begin{array}{l}\text { Choerodon schoenleinii (Valenciennes, } \\
\text { 1839) }\end{array}$ & RFA & & NT & 3.4 & $\mathrm{r} 1$ \\
\hline \multicolumn{6}{|l|}{ Genus Cirrhilabrus } \\
\hline $\begin{array}{l}\text { Cirrhilabrus melanomarginatus Randall \& } \\
\text { Shen, } 1978\end{array}$ & RFA & & LC & 3.4 & $\mathrm{r} 6, \mathrm{r} 11$ \\
\hline Cirrhilabrus solorensis Bleeker, 1853 & RFA & & & 3.4 & $\mathrm{r} 1$ \\
\hline Cirrhilabrus brunneus Allen, 2006 & & & & 3.2 & $\mathrm{r} 1$ \\
\hline
\end{tabular}




\begin{tabular}{|c|c|c|c|c|c|}
\hline $\begin{array}{l}\text { Subclass, Order, Family, Genus, and } \\
\text { species }\end{array}$ & $\begin{array}{l}\text { Habitat } \\
\text { types }\end{array}$ & Threat to humans & $\begin{array}{l}\text { IUCN } \\
\text { status }\end{array}$ & $\begin{array}{l}\text { Trophic } \\
\text { level }\end{array}$ & $\begin{array}{l}\text { Other } \\
\text { remarks }\end{array}$ \\
\hline Cirrhilabrus cyanopleura (Bleeker, 1851) & RFA & & & 3.4 & r9,r11 \\
\hline${ }^{*}$ Cirrhilabrus exquisitus Smith, 1957 & RFA & & & 3.4 & $r 10, r 11$ \\
\hline \multicolumn{6}{|l|}{ Genus Coris } \\
\hline Coris gaimard (Quoy \& Gaimard, 1824) & RFA & $\begin{array}{l}\text { Reports of ciguatera } \\
\text { poisoning }\end{array}$ & LC & 3.5 & $\mathrm{r} 1, \mathrm{r} 11$ \\
\hline${ }^{*}$ Coris aygula Lacepède, 1801 & RFA & & LC & 3.7 & r11 \\
\hline Coris dorsomacula Fowler, 1908 & RFA & & LC & 3.5 & r9,r10,r11 \\
\hline \multicolumn{6}{|l|}{ Genus Cymolutes } \\
\hline $\begin{array}{l}\text { Cymolutes lecluse (Quoy \& Gaimard, } \\
\text { 1824) }\end{array}$ & RFA & & LC & 4.2 & $\mathrm{r} 1$ \\
\hline \multicolumn{6}{|l|}{ Genus Epibulus } \\
\hline Epibulus insidiator (Pallas, 1770) & RFA & $\begin{array}{l}\text { Reports of ciguatera } \\
\text { poisoning }\end{array}$ & LC & 4.0 & $\mathrm{r} 1, \mathrm{r} 8, \mathrm{r} 11$ \\
\hline \multicolumn{6}{|l|}{ Genus Gomphosus } \\
\hline Gomphosus varius Lacepède, 1801 & RFA & & LC & 3.7 & $\mathrm{r} 1, \mathrm{r} 11$ \\
\hline \multicolumn{6}{|l|}{ Genus Halichoeres } \\
\hline Halichoeres biocellatus Schultz, 1960 & RFA & & LC & 3.4 & $\mathrm{r} 11$ \\
\hline Halichoeres chrysus Randall, 1981 & RFA & & LC & 3.4 & r6 \\
\hline Halichoeres hartzfeldii (Bleeker, 1852) & RFA & & LC & 3.5 & $\mathrm{r} 1$ \\
\hline Halichoeres hortulanus (Lacepède, 1801) & RFA & & LC & 3.4 & $\mathrm{r} 1, \mathrm{r} 6, \mathrm{r} 8, \mathrm{r} 11$ \\
\hline $\begin{array}{l}\text { Halichoeres margaritaceus (Valenciennes, } \\
\text { 1839) }\end{array}$ & RFA & & LC & 3.7 & $\mathrm{r} 1, \mathrm{r} 11$ \\
\hline Halichoeres marginatus Rüppell, 1835 & RFA & & LC & 3.2 & $\mathrm{r} 1, \mathrm{r} 11$ \\
\hline Halichoeres prosopeion (Bleeker, 1853) & RFA & & LC & 3.5 & r3,r11 \\
\hline $\begin{array}{l}\text { Halichoeres nigrescens (Bloch \& } \\
\text { Schneider, 1801) }\end{array}$ & RFA & & LC & 3.4 & $\mathrm{r} 1, \mathrm{r} 11$ \\
\hline $\begin{array}{l}\text { *Halichoeres melasmapomus Randall, } \\
1981\end{array}$ & RFA & & LC & 3.5 & $\mathrm{r} 11$ \\
\hline $\begin{array}{l}\text { Halichoeres trimaculatus (Quoy \& } \\
\text { Gaimard, 1834) }\end{array}$ & RFA & & LC & 3.5 & r1,r8.r11 \\
\hline *Halichoeres zeylonicus (Bennett, 1833) & RFA & & LC & 3.5 & $\mathrm{r} 11$ \\
\hline Genus Hemigymnus & & & & & \\
\hline
\end{tabular}




\begin{tabular}{|c|c|c|c|c|c|}
\hline $\begin{array}{l}\text { Subclass, Order, Family, Genus, and } \\
\text { species }\end{array}$ & $\begin{array}{l}\text { Habitat } \\
\text { types }\end{array}$ & Threat to humans & $\begin{array}{l}\text { IUCN } \\
\text { status }\end{array}$ & $\begin{array}{l}\text { Trophic } \\
\text { level }\end{array}$ & $\begin{array}{l}\text { Other } \\
\text { remarks }\end{array}$ \\
\hline Hemigymnus fasciatus (Bloch, 1792) & RFA & & LC & 3.5 & $r 1, r 6, r 11$ \\
\hline Hemigymnus melapterus (Bloch, 1791) & RFA & & LC & 3.6 & $r 1, r 6, r 11$ \\
\hline \multicolumn{6}{|l|}{ Genus Hologymnosus } \\
\hline $\begin{array}{l}\text { Hologymnosus annulatus (Lacepède, } \\
\text { 1801) }\end{array}$ & RFA & & LC & 4.2 & $\mathrm{r} 1, \mathrm{r} 11$ \\
\hline Hologymnosus doliatus (Lacepède, 1801) & RFA & & LC & 3.8 & $\mathrm{r} 9, \mathrm{r} 10, \mathrm{r} 11$ \\
\hline \multicolumn{6}{|l|}{ Genus Iniistius } \\
\hline Iniistius aneitensis (Günther, 1862) & RFA & & LC & 3.5 & r1 \\
\hline Iniistius melanopus (Bleeker, 1857) & RFA & & LC & 3.5 & $\mathrm{r} 1$ \\
\hline Iniistius pavo (Valenciennes, 1840) & RFA & & LC & 3.5 & $\mathrm{r} 1$ \\
\hline \multicolumn{6}{|l|}{ Genus Labrichthys } \\
\hline Labrichthys unilineatus (Guichenot, 1847) & RFA & & LC & 3.3 & $r 1, r 6$ \\
\hline \multicolumn{6}{|l|}{ Genus Labroides } \\
\hline Labroides bicolor Fowler \& Bean, 1928 & RFA & & LC & 4.0 & r6,r11 \\
\hline Labroides dimidiatus (Valenciennes, 1839) & RFA & & LC & 3.5 & $\mathrm{r} 1, \mathrm{r} 6, \mathrm{r} 11$ \\
\hline \multicolumn{6}{|l|}{ Genus Labropsis } \\
\hline Labropsis manabei Schmidt, 1931 & RFA & & LC & 3.3 & $\mathrm{r} 1, \mathrm{r} 6$ \\
\hline \multicolumn{6}{|l|}{ Genus Macropharyngodon } \\
\hline $\begin{array}{l}\text { Macropharyngodon meleagris } \\
\text { (Valenciennes, 1839) }\end{array}$ & RFA & & LC & 3.1 & $r 1, r 8, r 11$ \\
\hline \multicolumn{6}{|l|}{ Genus Novaculichthys } \\
\hline $\begin{array}{l}\text { Novaculichthys taeniourus (Lacepède, } \\
\text { 1801) }\end{array}$ & RFA & & LC & 3.3 & $\mathrm{r} 1$ \\
\hline \multicolumn{6}{|l|}{ Genus Oxycheilinus } \\
\hline Oxycheilinus celebicus (Bleeker, 1853) & RFA & & LC & 3.8 & $\mathrm{r} 1, \mathrm{r} 11$ \\
\hline Oxycheilinus digramma (Lacepède, 1801) & RFA & & LC & 3.7 & $\mathrm{r} 1, \mathrm{r} 11$ \\
\hline Oxycheilinus mentalis (Rüppell, 1828) & RFA & & LC & 3.8 & $\mathrm{r} 1$ \\
\hline Oxycheilinus orientalis (Günther, 1862) & RFA & & LC & 3.8 & $\mathrm{r} 1$ \\
\hline Oxycheilinus unifasciatus (Streets, 1877) & RFA & $\begin{array}{l}\text { Reports of ciguatera } \\
\text { poisoning }\end{array}$ & LC & 4.1 & $\mathrm{r} 6, \mathrm{r} 11$ \\
\hline
\end{tabular}




\begin{tabular}{|c|c|c|c|c|c|}
\hline $\begin{array}{l}\text { Subclass, Order, Family, Genus, and } \\
\text { species }\end{array}$ & $\begin{array}{l}\text { Habitat } \\
\text { types }\end{array}$ & Threat to humans & $\begin{array}{l}\text { IUCN } \\
\text { status }\end{array}$ & $\begin{array}{l}\text { Trophic } \\
\text { level }\end{array}$ & $\begin{array}{l}\text { Other } \\
\text { remarks }\end{array}$ \\
\hline $\begin{array}{l}\text { Oxycheilinus bimaculatus (Valenciennes, } \\
\text { 1840) }\end{array}$ & RFA & & LC & 3.5 & r9,r11 \\
\hline \multicolumn{6}{|l|}{ Genus Pseudocheilinus } \\
\hline $\begin{array}{l}\text { Pseudocheilinus evanidus Jordan \& } \\
\text { Evermann, } 1903\end{array}$ & RFA & & LC & 3.5 & $\mathrm{r} 11$ \\
\hline $\begin{array}{l}\text { Pseudocheilinus hexataenia (Bleeker, } \\
\text { 1857) }\end{array}$ & RFA & & LC & 3.2 & $\mathrm{r} 1, \mathrm{r} 6$ \\
\hline \multicolumn{6}{|l|}{ Genus Pseudocoris } \\
\hline Pseudocoris yamashiroi (Schmidt, 1931) & RFA & & LC & 3.4 & r1 \\
\hline \multicolumn{6}{|l|}{ Genus Pteragogus } \\
\hline $\begin{array}{l}\text { Pteragogus flagellifer (Valenciennes, } \\
\text { 1839) }\end{array}$ & RFA & & LC & 3.5 & $\mathrm{r} 1$ \\
\hline \multicolumn{6}{|l|}{ Genus Stethojulis } \\
\hline $\begin{array}{l}\text { Stethojulis balteata (Quoy \& Gaimard, } \\
\text { 1824) }\end{array}$ & RFA & & LC & 3.5 & r1 \\
\hline Stethojulis bandanensis (Bleeker, 1851) & RFA & & LC & 3.2 & $r 1, r 11$ \\
\hline Stethojulis interrupta (Bleeker, 1851) & RFA & & LC & 3.4 & $\mathrm{r} 1, \mathrm{r} 8$ \\
\hline Stethojulis strigiventer (Bennett, 1833) & RFA & & LC & 3.1 & $\mathrm{r} 1, \mathrm{r} 11$ \\
\hline \multicolumn{6}{|l|}{ Genus Thalassoma } \\
\hline $\begin{array}{l}\text { Thalassoma cupido (Temminck \& } \\
\text { Schlegel, 1845) }\end{array}$ & DEM & & LC & 3.5 & r6 \\
\hline Thalassoma hardwicke (Bennett, 1830) & RFA & & LC & 3.5 & $r 1, r 6, r 8, r 11$ \\
\hline Thalassoma lunare (Linnaeus, 1758) & RFA & & LC & 3.5 & $r 1, r 6, r 11$ \\
\hline Thalassoma purpureum (Forsskål, 1775) & RFA & & LC & 3.8 & $\mathrm{r} 1, \mathrm{r} 6$ \\
\hline $\begin{array}{l}\text { Thalassoma quinquevittatum (Lay \& } \\
\text { Bennett, 1839) }\end{array}$ & RFA & & LC & 3.6 & $r 1, r 6, r 11$ \\
\hline Thalassoma trilobatum (Lacepède, 1801) & RFA & & LC & 3.8 & $\mathrm{r} 1, \mathrm{r} 6$ \\
\hline $\begin{array}{l}\text { Thalassoma lutescens (Lay \& Bennett, } \\
\text { 1839) }\end{array}$ & RFA & & LC & 3.7 & r9,r10,r11 \\
\hline $\begin{array}{l}\text { Thalassoma amblycephalum (Bleeker, } \\
\text { 1856) }\end{array}$ & RFA & & LC & 3.1 & $\mathrm{r} 9, \mathrm{r} 10, \mathrm{r} 11$ \\
\hline Family Scaridae & & & & & \\
\hline
\end{tabular}




\begin{tabular}{|c|c|c|c|c|c|}
\hline $\begin{array}{l}\text { Subclass, Order, Family, Genus, and } \\
\text { species }\end{array}$ & $\begin{array}{l}\text { Habitat } \\
\text { types }\end{array}$ & Threat to humans & $\begin{array}{l}\text { IUCN } \\
\text { status }\end{array}$ & $\begin{array}{l}\text { Trophic } \\
\text { level }\end{array}$ & $\begin{array}{l}\text { Other } \\
\text { remarks }\end{array}$ \\
\hline \multicolumn{6}{|l|}{ Genus Calotomus } \\
\hline Calotomus japonicus (Valenciennes, 1840) & RFA & & LC & 2.0 & $\mathrm{r} 1$ \\
\hline $\begin{array}{l}\text { Calotomus spinidens (Quoy \& Gaimard, } \\
\text { 1824) }\end{array}$ & RFA & & LC & 2.0 & $\mathrm{r} 1$ \\
\hline \multicolumn{6}{|l|}{ Genus Cetoscarus } \\
\hline Cetoscarus bicolor (Rüppell, 1829) & RFA & & LC & 2.0 & $\mathrm{r} 1, \mathrm{r} 11$ \\
\hline \multicolumn{6}{|l|}{ Genus Chlorurus } \\
\hline Chlorurus microrhinos (Bleeker, 1854) & RFA & $\begin{array}{l}\text { Reports of ciguatera } \\
\text { poisoning }\end{array}$ & LC & 2.6 & $\mathrm{r} 1$ \\
\hline Chlorurus sordidus (Forsskål, 1775) & RFA; OD & $\begin{array}{l}\text { Reports of ciguatera } \\
\text { poisoning }\end{array}$ & LC & 2.6 & $\mathrm{r} 1, \mathrm{r} 6, \mathrm{r} 7, \mathrm{r} 8, \mathrm{r} 11$ \\
\hline Chlorurus gibbus (Rüppell, 1829) & RFA & $\begin{array}{l}\text { Reports of ciguatera } \\
\text { poisoning }\end{array}$ & LC & 2.0 & $\mathrm{r} 1$ \\
\hline Chlorurus bleekeri (de Beaufort, 1940) & RFA & & LC & 2.0 & r10,r11 \\
\hline Chlorurus japanensis (Bloch, 1789) & RFA & & LC & 2.0 & r6,r9,r10,r11 \\
\hline \multicolumn{6}{|l|}{ Genus Hipposcarus } \\
\hline $\begin{array}{l}\text { Hipposcarus longiceps (Valenciennes, } \\
\text { 1840) }\end{array}$ & RFA & & LC & 2.0 & r1 \\
\hline \multicolumn{6}{|l|}{ Genus Leptoscarus } \\
\hline $\begin{array}{l}\text { Leptoscarus vaigiensis (Quoy \& Gaimard, } \\
1824 \text { ) }\end{array}$ & RFA & & LC & 2.0 & r1 \\
\hline \multicolumn{6}{|l|}{ Genus Scarus } \\
\hline Scarus dimidiatus Bleeker, 1859 & RFA & & LC & 2.0 & $\mathrm{r} 1, \mathrm{r} 8, \mathrm{r} 11$ \\
\hline Scarus ferrugineus Forsskål, 1775 & RFA & & LC & 2.0 & $\mathrm{r} 1, \mathrm{r} 7$ \\
\hline Scarus festivus Valenciennes, 1840 & RFA & & LC & 2.0 & $\mathrm{r} 1, \mathrm{r} 11$ \\
\hline Scarus forsteni (Bleeker, 1861) & RFA & & LC & 2.0 & $r 1, r 8, r 11$ \\
\hline Scarus frenatus Lacepède, 1802 & RFA & & LC & 2.0 & $\mathrm{r} 1, \mathrm{r} 11$ \\
\hline Scarus ghobban Forsskål, 1775 & RFA & & LC & 2.0 & r1 \\
\hline Scarus globiceps Valenciennes, 1840 & RFA & & LC & 2.0 & $\mathrm{r} 1, \mathrm{r} 11$ \\
\hline Scarus niger Forsskål, 1775 & RFA & & LC & 2.0 & $\mathrm{r} 1, \mathrm{r} 11$ \\
\hline Scarus oviceps Valenciennes, 1840 & RFA & & LC & 2.0 & $\mathrm{r} 1, \mathrm{r} 7, \mathrm{r} 11$ \\
\hline
\end{tabular}




\begin{tabular}{|c|c|c|c|c|c|}
\hline $\begin{array}{l}\text { Subclass, Order, Family, Genus, and } \\
\text { species }\end{array}$ & $\begin{array}{l}\text { Habitat } \\
\text { types }\end{array}$ & Threat to humans & $\begin{array}{l}\text { IUCN } \\
\text { status }\end{array}$ & $\begin{array}{l}\text { Trophic } \\
\text { level }\end{array}$ & $\begin{array}{l}\text { Other } \\
\text { remarks }\end{array}$ \\
\hline Scarus prasiognathos Valenciennes, 1840 & RFA & & LC & 2.0 & $\mathrm{r} 1, \mathrm{r} 7$ \\
\hline Scarus psittacus Forsskål, 1775 & RFA & & LC & 2.0 & $\mathrm{r} 1, \mathrm{r} 7, \mathrm{r} 11$ \\
\hline Scarus rivulatus Valenciennes, 1840 & RFA & & LC & 2.0 & $\mathrm{r} 1, \mathrm{r} 11$ \\
\hline Scarus rubroviolaceus Bleeker, 1847 & RFA & & LC & 2.0 & $\mathrm{r} 1$ \\
\hline Scarus scaber Valenciennes, 1840 & RFA & & LC & 2.0 & $\mathrm{r} 1$ \\
\hline Scarus tricolor Bleeker, 1847 & RFA & & LC & 2.0 & $\mathrm{r} 1, \mathrm{r} 7$ \\
\hline Scarus schlegeli (Bleeker, 1861) & RFA & & LC & 2.0 & r9.r10,r11 \\
\hline \multicolumn{6}{|l|}{ Family Pinguipedidae } \\
\hline \multicolumn{6}{|l|}{ Genus Parapercis } \\
\hline Parapercis clathrata Ogilby, 1910 & RFA & & & 3.6 & $\mathrm{r} 1, \mathrm{r} 11$ \\
\hline Parapercis cylindrica (Bloch, 1792) & RFA & & & 3.0 & $\mathrm{r} 1, \mathrm{r} 8, \mathrm{r} 11$ \\
\hline $\begin{array}{l}\text { Parapercis pacifica Imamura \& Yoshino, } \\
2007\end{array}$ & RFA & & & 3.6 & $\mathrm{r} 1, \mathrm{r} 6, \mathrm{r} 8, \mathrm{r} 11$ \\
\hline *Parapercis millepunctata (Günther, 1860) & RFA & & & 3.5 & $\mathrm{r} 11$ \\
\hline *Parapercis xanthozona (Bleeker, 1849) & RFA & & LC & 3.6 & $\mathrm{r} 11$ \\
\hline Parapercis hexophtalma (Cuvier, 1829) & RFA & & & 3.6 & r11 \\
\hline \multicolumn{6}{|l|}{ Family Uranoscopidae } \\
\hline \multicolumn{6}{|l|}{ Genus Uranoscopidae } \\
\hline Uranoscopus japonicus Houttuyn, 1782 & BAD & Venomous & LC & 4.0 & $\mathrm{r} 1$ \\
\hline \multicolumn{6}{|l|}{ Family Tripterygiidae } \\
\hline \multicolumn{6}{|l|}{ Genus Helcogramma } \\
\hline *Helcogramma chica Rosenblatt, 1960 & RFA & & LC & 3.0 & r11 \\
\hline \multicolumn{6}{|l|}{ Family Blenniidae } \\
\hline \multicolumn{6}{|l|}{ Genus Aspidontus } \\
\hline Aspidontus tractus Fowler, 1903 & RFA & & & 2.9 & $\mathrm{r} 1$ \\
\hline $\begin{array}{l}\text { Aspidontus taeniatus Quoy \& Gaimard, } \\
1834\end{array}$ & & & & 3.8 & r8,r11 \\
\hline \multicolumn{6}{|l|}{ Genus Blenniella } \\
\hline $\begin{array}{l}\text { Blenniella periophthalmus (Valenciennes, } \\
\text { 1836) }\end{array}$ & RFA & & LC & 3.3 & $\mathrm{r} 1$ \\
\hline Genus Cirripectes & & & & & \\
\hline
\end{tabular}




\begin{tabular}{|c|c|c|c|c|c|}
\hline $\begin{array}{l}\text { Subclass, Order, Family, Genus, and } \\
\text { species }\end{array}$ & $\begin{array}{l}\text { Habitat } \\
\text { types }\end{array}$ & Threat to humans & $\begin{array}{l}\text { IUCN } \\
\text { status }\end{array}$ & $\begin{array}{l}\text { Trophic } \\
\text { level }\end{array}$ & $\begin{array}{l}\text { Other } \\
\text { remarks }\end{array}$ \\
\hline $\begin{array}{l}\text { Cirripectes castaneus (Valenciennes, } \\
1836 \text { ) }\end{array}$ & RFA & & LC & 2.0 & $\mathrm{r} 6, \mathrm{r} 11$ \\
\hline $\begin{array}{l}\text { Cirripectes variolosus (Valenciennes, } \\
\text { 1836) }\end{array}$ & RFA & & LC & 2.0 & $\mathrm{r} 1$ \\
\hline \multicolumn{6}{|l|}{ Genus Entomacrodus } \\
\hline $\begin{array}{l}\text { Entomacrodus caudofasciatus (Regan, } \\
\text { 1909) }\end{array}$ & RFA & & LC & 2.0 & $\mathrm{r} 1$ \\
\hline \multicolumn{6}{|l|}{ Genus Istiblennius } \\
\hline $\begin{array}{l}\text { Istiblennius dussumieri (Valenciennes, } \\
1836 \text { ) }\end{array}$ & RFA & & LC & 2.0 & $\mathrm{r} 1$ \\
\hline $\begin{array}{l}\text { Istiblennius edentulus (Forster \& } \\
\text { Schneider, 1801) }\end{array}$ & RFA & & LC & 2.0 & $\mathrm{r} 1$ \\
\hline Istiblennius lineatus (Valenciennes, 1836) & RFA & & LC & 2.0 & $\mathrm{r} 1$ \\
\hline \multicolumn{6}{|l|}{ Genus Meiacanthus } \\
\hline Meiacanthus atrodorsalis (Günther, 1877) & RFA & Venomous & LC & 3.5 & r6 \\
\hline \multicolumn{6}{|l|}{ Genus Plagiotremus } \\
\hline $\begin{array}{l}\text { Plagiotremus rhinorhynchos (Bleeker, } \\
\text { 1852) }\end{array}$ & RFA & & LC & 4.5 & r6,r11 \\
\hline Plagiotremus tapeinosoma (Bleeker, 1857) & RFA & Other & LC & 3.8 & r6,r11 \\
\hline \multicolumn{6}{|l|}{ Genus Salarias } \\
\hline Salarias fasciatus (Bloch, 1786) & RFA & & LC & 2.0 & $\mathrm{r} 1$ \\
\hline \multicolumn{6}{|l|}{ Genus praealticus } \\
\hline $\begin{array}{l}\text { Praealticus margaritatus (Kendall \& } \\
\text { Radcliffe, 1912) }\end{array}$ & PN & & LC & 2.0 & $\mathrm{r} 1$ \\
\hline \multicolumn{6}{|l|}{ Family Ptereleotridae } \\
\hline \multicolumn{6}{|l|}{ Genus Nemateleotris } \\
\hline Nemateleotris magnifica Fowler, 1938 & RFA & & LC & 3.1 & $\mathrm{r} 6, \mathrm{r} 11$ \\
\hline \multicolumn{6}{|l|}{ Genus Ptereleotris } \\
\hline $\begin{array}{l}\text { Ptereleotris evides (Jordan \& Hubbs, } \\
\text { 1925) }\end{array}$ & RFA & & LC & 3.4 & $r 6, r 11$ \\
\hline \multicolumn{6}{|l|}{ Family Gobiidae } \\
\hline Genus Acentrogobius & & & & & \\
\hline
\end{tabular}




\begin{tabular}{|c|c|c|c|c|c|}
\hline $\begin{array}{l}\text { Subclass, Order, Family, Genus, and } \\
\text { species }\end{array}$ & $\begin{array}{l}\text { Habitat } \\
\text { types }\end{array}$ & Threat to humans & $\begin{array}{l}\text { IUCN } \\
\text { status }\end{array}$ & $\begin{array}{l}\text { Trophic } \\
\text { level }\end{array}$ & $\begin{array}{l}\text { Other } \\
\text { remarks }\end{array}$ \\
\hline $\begin{array}{l}\text { Acentrogobius caninus (Valenciennes, } \\
\text { 1837) }\end{array}$ & $\begin{array}{l}\text { DEM; } \\
\text { AMP }\end{array}$ & Poisonous to eat & LC & 3.5 & $\mathrm{r} 1$ \\
\hline \multicolumn{6}{|l|}{ Genus Amblygobius } \\
\hline $\begin{array}{l}\text { Amblygobius albimaculatus (Rüppell, } \\
\text { 1830) }\end{array}$ & RFA & & & 2.6 & $\mathrm{r} 1$ \\
\hline *Amblygobius nocturnus (Herre, 1945) & RFA & & & 2.7 & $\mathrm{r} 11$ \\
\hline $\begin{array}{l}\text { Amblygobius phalaena (Valenciennes, } \\
\text { 1837) }\end{array}$ & RFA & & & 3.6 & r9,r11 \\
\hline \multicolumn{6}{|l|}{ Genus Asterropteryx } \\
\hline Asterropteryx semipunctata Rüppell, 1830 & RFA & & & 2.4 & $\mathrm{r} 1$ \\
\hline \multicolumn{6}{|l|}{ Genus Bathygobius } \\
\hline Bathygobius fuscus (Rüppell, 1830) & RFA & & LC & 3.4 & $\mathrm{r} 1$ \\
\hline \multicolumn{6}{|l|}{ Genus Callogobius } \\
\hline Callogobius sclateri (Steindachner, 1879) & RFA & & & 3.3 & $\mathrm{r} 1$ \\
\hline \multicolumn{6}{|l|}{ Genus Ctenogobiops } \\
\hline $\begin{array}{l}\text { Ctenogobiops feroculus Lubbock \& } \\
\text { Polunin, } 1977\end{array}$ & RFA & & LC & 3.4 & r8 \\
\hline \multicolumn{6}{|l|}{ Genus Eviota } \\
\hline Eviota abax (Jordan \& Snyder, 1901) & RFA & & & 3.2 & $\mathrm{r} 1$ \\
\hline Eviota prasites Jordan \& Seale, 1906 & RFA & & LC & 3.1 & r9,r11 \\
\hline \multicolumn{6}{|l|}{ Genus Gobiodon } \\
\hline Gobiodon erythrospilus Bleeker, 1875 & RFA & & & 3.4 & $\mathrm{r} 1$ \\
\hline Gobiodon multilineatus Wu, 1979 & RFA & & & 3.2 & $\mathrm{r} 1$ \\
\hline Gobiodon oculolineatus Wu, 1979 & RFA & & & 3.2 & $\mathrm{r} 1$ \\
\hline $\begin{array}{l}\text { Gobiodon okinawae Sawada, Arai \& Abe, } \\
1972\end{array}$ & RFA & & & 3.2 & $\mathrm{r} 1$ \\
\hline $\begin{array}{l}\text { Gobiodon quinquestrigatus (Valenciennes, } \\
\text { 1837) }\end{array}$ & RFA & & & 3.4 & $\mathrm{r} 1$ \\
\hline \multicolumn{6}{|l|}{ Genus Istigobius } \\
\hline Istigobius ornatus (Rüppell, 1830) & RFA & & LC & 3.5 & $r 1$ \\
\hline Genus Paragobiodon & & & & & \\
\hline
\end{tabular}




\begin{tabular}{|c|c|c|c|c|c|}
\hline $\begin{array}{l}\text { Subclass, Order, Family, Genus, and } \\
\text { species }\end{array}$ & $\begin{array}{l}\text { Habitat } \\
\text { types }\end{array}$ & Threat to humans & $\begin{array}{l}\text { IUCN } \\
\text { status }\end{array}$ & $\begin{array}{l}\text { Trophic } \\
\text { level }\end{array}$ & $\begin{array}{l}\text { Other } \\
\text { remarks }\end{array}$ \\
\hline $\begin{array}{l}\text { Paragobiodon echinocephalus (Rüppell, } \\
\text { 1830) }\end{array}$ & RFA & & LC & 3.2 & $\mathrm{r} 1$ \\
\hline $\begin{array}{l}\text { Paragobiodon melanosoma (Bleeker, } \\
\text { 1853) }\end{array}$ & RFA & & LC & 3.1 & $\mathrm{r} 1$ \\
\hline $\begin{array}{l}\text { Paragobiodon xanthosoma (Bleeker, } \\
\text { 1853) }\end{array}$ & RFA & & LC & 3.2 & $\mathrm{r} 1$ \\
\hline \multicolumn{6}{|l|}{ Genus Priolepis } \\
\hline Priolepis semidoliata (Valenciennes, 1837) & RFA & & LC & 3.1 & $\mathrm{r} 1$ \\
\hline \multicolumn{6}{|l|}{ Genus Valenciennea } \\
\hline $\begin{array}{l}\text { Valenciennea longipinnis (Lay \& Bennett, } \\
\text { 1839) }\end{array}$ & RFA & & & 3.0 & r1 \\
\hline Valenciennea strigata (Broussonet, 1782) & RFA & & & 4.0 & $\mathrm{r} 1, \mathrm{r} 6, \mathrm{r} 11$ \\
\hline \multicolumn{6}{|l|}{ Family Ephippidae } \\
\hline \multicolumn{6}{|l|}{ Genus Platax } \\
\hline Platax orbicularis (Forsskål, 1775) & RFA & & LC & 3.3 & r2 \\
\hline Platax teira (Forsskål, 1775) & $\begin{array}{l}\text { RFA; } \\
\text { AMP }\end{array}$ & & LC & 4.0 & r2 \\
\hline \multicolumn{6}{|l|}{ Family Siganidae } \\
\hline \multicolumn{6}{|l|}{ Genus Siganus } \\
\hline $\begin{array}{l}\text { Siganus argenteus (Quoy \& Gaimard, } \\
\text { 1825) }\end{array}$ & RFA & Venomous & LC & 2.0 & $\mathrm{r} 1$ \\
\hline Siganus canaliculatus (Park, 1797) & RFA; OD & Venomous & LC & 2.8 & $\mathrm{r} 1$ \\
\hline Siganus corallinus (Valenciennes, 1835) & RFA & Venomous & LC & 2.0 & r1 \\
\hline Siganus fuscescens (Houttuyn, 1782) & RFA & Venomous & LC & 2.0 & $\mathrm{r} 1$ \\
\hline Siganus guttatus (Bloch, 1787) & RFA & Venomous & LC & 2.7 & $\mathrm{r} 1, \mathrm{r} 7$ \\
\hline Siganus puellus (Schlegel, 1852) & RFA & Venomous & LC & 3.0 & $\mathrm{r} 1, \mathrm{r} 11$ \\
\hline $\begin{array}{l}\text { Siganus punctatus (Schneider \& Forster, } \\
\text { 1801) }\end{array}$ & RFA & Venomous & LC & 2.0 & $\mathrm{r} 1, \mathrm{r} 11$ \\
\hline Siganus spinus (Linnaeus, 1758) & RFA & Venomous & LC & 2.0 & $\mathrm{r} 1$ \\
\hline Siganus virgatus (Valenciennes, 1835) & RFA & Venomous & LC & 2.7 & r2 \\
\hline $\begin{array}{l}\text { Siganus vulpinus (Schlegel \& Müller, } \\
1845 \text { ) }\end{array}$ & RFA & Venomous & LC & 2.7 & $\mathrm{r} 1, \mathrm{r} 6$ \\
\hline
\end{tabular}




\begin{tabular}{|c|c|c|c|c|c|}
\hline $\begin{array}{l}\text { Subclass, Order, Family, Genus, and } \\
\text { species }\end{array}$ & $\begin{array}{l}\text { Habitat } \\
\text { types }\end{array}$ & Threat to humans & $\begin{array}{l}\text { IUCN } \\
\text { status }\end{array}$ & $\begin{array}{l}\text { Trophic } \\
\text { level }\end{array}$ & $\begin{array}{l}\text { Other } \\
\text { remarks }\end{array}$ \\
\hline \multicolumn{6}{|l|}{ Family Acanthuridae } \\
\hline \multicolumn{6}{|l|}{ Genus Ctenochaetus } \\
\hline $\begin{array}{l}\text { Ctenochaetus striatus (Quoy \& Gaimard, } \\
\text { 1825) }\end{array}$ & RFA & $\begin{array}{l}\text { Reports of ciguatera } \\
\text { poisoning }\end{array}$ & LC & 2.0 & $\mathrm{r} 1, \mathrm{r} 6, \mathrm{r} 8, \mathrm{r} 11$ \\
\hline $\begin{array}{l}\text { Ctenochaetus cyanocheilus Randall \& } \\
\text { Clements, } 2001\end{array}$ & RFA & & LC & 2.0 & r9,r11 \\
\hline \multicolumn{6}{|l|}{ Genuus Naso } \\
\hline Naso annulatus (Quoy \& Gaimard, 1825) & RFA & $\begin{array}{l}\text { Reports of ciguatera } \\
\text { poisoning }\end{array}$ & LC & 2.1 & r3 \\
\hline Naso brachycentron (Valenciennes, 1835) & RFA & & LC & 2.7 & r2 \\
\hline Naso brevirostris (Cuvier, 1829) & RFA & $\begin{array}{l}\text { Reports of ciguatera } \\
\text { poisoning }\end{array}$ & LC & 2.2 & $\mathrm{r} 1, \mathrm{r} 11$ \\
\hline Naso hexacanthus (Bleeker, 1855) & RFA & & LC & 3.1 & $\mathrm{r} 1, \mathrm{r} 7, \mathrm{r} 11$ \\
\hline Naso lituratus (Forster, 1801) & RFA & Venomous & LC & 2.3 & $\mathrm{r} 1, \mathrm{r} 6$ \\
\hline Naso lopezi Herre, 1927 & RFA & & LC & 2.9 & r2 \\
\hline Naso thynnoides (Cuvier, 1829) & RFA & & LC & 3.0 & $\mathrm{r} 1, \mathrm{r} 6$ \\
\hline Naso unicornis (Forsskål, 1775) & RFA & $\begin{array}{l}\text { Reports of ciguatera } \\
\text { poisoning }\end{array}$ & LC & 2.2 & $\mathrm{r} 1$ \\
\hline Naso vlamingii (Valenciennes, 1835) & RFA & & LC & 2.2 & $\mathrm{r} 1$ \\
\hline \multicolumn{6}{|l|}{ Genus Prionurus } \\
\hline Prionurus scalprum Valenciennes, 1835 & RFA & Venomous & & 2.0 & $\mathrm{r} 1, \mathrm{r} 7$ \\
\hline \multicolumn{6}{|l|}{ Genus Zebrasoma } \\
\hline Zebrasoma flavescens (Bennett, 1828) & RFA & & LC & 2.0 & $\mathrm{r} 1, \mathrm{r} 8$ \\
\hline Zebrasoma scopas (Cuvier, 1829) & RFA & & LC & 2.0 & r6,r8,r11 \\
\hline Zebrasoma velifer (Bloch, 1795) & RFA & & LC & 2.0 & $r 1, r 6, r 8, r 11$ \\
\hline \multicolumn{6}{|l|}{ Family Zanclidae } \\
\hline \multicolumn{6}{|l|}{ Genus Zanclus } \\
\hline Zanclus cornutus (Linnaeus, 1758) & RFA & & LC & 2.5 & $r 1, r 6, r 8, r 11$ \\
\hline \multicolumn{6}{|l|}{ Family Acanthuridae } \\
\hline Genus Acanthurus & & & & & \\
\hline
\end{tabular}




\begin{tabular}{|c|c|c|c|c|c|}
\hline $\begin{array}{l}\text { Subclass, Order, Family, Genus, and } \\
\text { species }\end{array}$ & $\begin{array}{l}\text { Habitat } \\
\text { types }\end{array}$ & Threat to humans & $\begin{array}{l}\text { IUCN } \\
\text { status }\end{array}$ & $\begin{array}{l}\text { Trophic } \\
\text { level }\end{array}$ & $\begin{array}{l}\text { Other } \\
\text { remarks }\end{array}$ \\
\hline $\begin{array}{l}\text { Acanthurus dussumieri Valenciennes, } \\
1835\end{array}$ & RFA & & LC & 2.0 & $\mathrm{r} 1$ \\
\hline Acanthurus gahhm (Forsskål, 1775) & RFA & & LC & 2.0 & r4 \\
\hline Acanthurus japonicus (Schmidt, 1931) & RFA & & LC & 2.0 & $\mathrm{r} 6, \mathrm{r} 11$ \\
\hline Acanthurus lineatus (Linnaeus, 1758) & RFA & Venomous & LC & 2.0 & $\mathrm{r} 1$ \\
\hline Acanthurus mata (Cuvier, 1829) & RFA & Venomous & LC & 2.5 & $\mathrm{r} 1$ \\
\hline Acanthurus nigricans (Linnaeus, 1758) & RFA & & LC & 2.0 & r2 \\
\hline Acanthurus nigrofuscus (Forsskål, 1775) & RFA & $\begin{array}{l}\text { Reports of ciguatera } \\
\text { poisoning }\end{array}$ & LC & 2.0 & $\mathrm{r} 1, \mathrm{r} 7, \mathrm{r} 11$ \\
\hline $\begin{array}{l}\text { Acanthurus olivaceus Bloch \& Schneider, } \\
1801\end{array}$ & RFA & & LC & 2.3 & $\mathrm{r} 1, \mathrm{r6}, \mathrm{r} 11$ \\
\hline Acanthurus thompsoni (Fowler, 1923) & RFA & & LC & 3.6 & $\mathrm{r} 1$ \\
\hline Acanthurus triostegus (Linnaeus, 1758) & RFA & $\begin{array}{l}\text { Reports of ciguatera } \\
\text { poisoning }\end{array}$ & LC & 2.8 & $r 1, r 6, r 11$ \\
\hline $\begin{array}{l}\text { Acanthurus xanthopterus Valenciennes, } \\
1835\end{array}$ & RFA & Venomous & LC & 2.9 & r2 \\
\hline \multicolumn{6}{|l|}{ Family Sphyraenidae } \\
\hline \multicolumn{6}{|l|}{ Genus Sphyraena } \\
\hline Sphyraena barracuda (Edwards, 1771) & RFA & Traumatogenic & LC & 4.5 & $\mathrm{r} 1, \mathrm{r} 7$ \\
\hline Sphyraena forsteri Cuvier, 1829 & RFA & $\begin{array}{l}\text { Reports of ciguatera } \\
\text { poisoning }\end{array}$ & & 4.4 & $\mathrm{r} 1, \mathrm{r} 7$ \\
\hline Sphyraena helleri Jenkins, 1901 & RFA & & & 4.5 & $\mathrm{r} 1$ \\
\hline Sphyraena obtusata Cuvier, 1829 & RFA & & & 4.5 & r1 \\
\hline \multicolumn{6}{|l|}{ Family Gempylidae } \\
\hline \multicolumn{6}{|l|}{ Genus Gempylus } \\
\hline Gempylus serpens Cuvier, 1829 & PEL; OD & & LC & 4.4 & $\mathrm{r} 1$ \\
\hline \multicolumn{6}{|l|}{ Genus Lepidocybium } \\
\hline $\begin{array}{l}\text { Lepidocybium flavobrunneum (Smith, } \\
\text { 1843) }\end{array}$ & BEP; OD & & LC & 4.3 & $\mathrm{r} 1$ \\
\hline \multicolumn{6}{|l|}{ Genus Promethichthys } \\
\hline $\begin{array}{l}\text { Promethichthys prometheus (Cuvier, } \\
\text { 1832) }\end{array}$ & BEP; OD & $\begin{array}{l}\text { Reports of ciguatera } \\
\text { poisoning }\end{array}$ & LC & 4.2 & $\mathrm{r} 1$ \\
\hline
\end{tabular}




\begin{tabular}{|c|c|c|c|c|c|}
\hline $\begin{array}{l}\text { Subclass, Order, Family, Genus, and } \\
\text { species }\end{array}$ & $\begin{array}{l}\text { Habitat } \\
\text { types }\end{array}$ & Threat to humans & $\begin{array}{l}\text { IUCN } \\
\text { status }\end{array}$ & $\begin{array}{l}\text { Trophic } \\
\text { level }\end{array}$ & $\begin{array}{l}\text { Other } \\
\text { remarks }\end{array}$ \\
\hline \multicolumn{6}{|l|}{ Genus Rexea } \\
\hline Rexea prometheoides (Bleeker, 1856) & BEP & & & 4.2 & r3 \\
\hline \multicolumn{6}{|l|}{ Genus Ruvettus } \\
\hline Ruvettus pretiosus Cocco, 1833 & BEP; OD & Poisonous to eat & LC & 4.2 & $\mathrm{r} 1, \mathrm{r} 7$ \\
\hline \multicolumn{6}{|l|}{ Genus Thyrsitoides } \\
\hline Thyrsitoides marleyi Fowler, 1929 & BEP & & & 4.2 & $\mathrm{r} 1, \mathrm{r} 7$ \\
\hline \multicolumn{6}{|l|}{ Family Scombridae } \\
\hline \multicolumn{6}{|l|}{ Genus Acanthocybium } \\
\hline Acanthocybium solandri (Cuvier, 1832) & PEL; OD & $\begin{array}{l}\text { Reports of ciguatera } \\
\text { poisoning }\end{array}$ & LC & 4.3 & r1 \\
\hline \multicolumn{6}{|l|}{ Genus Auxis } \\
\hline Auxis thazard (Lacepède, 1800) & PN; OD & & LC & 4.4 & $\mathrm{r} 1$ \\
\hline \multicolumn{6}{|l|}{ Genus Euthynnus } \\
\hline Euthynnus affinis (Cantor, 1849) & PN; OD & $\begin{array}{l}\text { Reports of ciguatera } \\
\text { poisoning }\end{array}$ & LC & 4.5 & $\mathrm{r} 1$ \\
\hline \multicolumn{6}{|l|}{ Genus Grammatorcynus } \\
\hline $\begin{array}{l}\text { Grammatorcynus bicarinatus (Quoy \& } \\
\text { Gaimard, 1825) }\end{array}$ & RFA; OD & & LC & 4.5 & $\mathrm{r} 1$ \\
\hline \multicolumn{6}{|l|}{ Genus Gymnosarda } \\
\hline Gymnosarda unicolor (Rüppell, 1836) & RFA; OD & $\begin{array}{l}\text { Reports of ciguatera } \\
\text { poisoning }\end{array}$ & LC & 4.5 & $\mathrm{r} 1$ \\
\hline \multicolumn{6}{|l|}{ Genus Katsuwonus } \\
\hline Katsuwonus pelamis (Linnaeus, 1758) & PEL; OD & $\begin{array}{l}\text { Reports of ciguatera } \\
\text { poisoning }\end{array}$ & LC & 4.4 & $\mathrm{r} 1$ \\
\hline \multicolumn{6}{|l|}{ Genus Rastrelliger } \\
\hline Rastrelliger kanagurta (Cuvier, 1816) & PN; OD & & & 3.2 & $\mathrm{r} 1$ \\
\hline \multicolumn{6}{|l|}{ Genus Thunnus } \\
\hline Thunnus albacares (Bonnaterre, 1788) & PEL; OD & & NT & 4.4 & r1 \\
\hline Thunnus obesus (Lowe, 1839) & PEL; OD & & VU & 4.5 & $\mathrm{r} 1$ \\
\hline \multicolumn{6}{|l|}{ Family Xiphiidae } \\
\hline Genus Xiphias & & & & & \\
\hline
\end{tabular}




\begin{tabular}{|c|c|c|c|c|c|}
\hline $\begin{array}{l}\text { Subclass, Order, Family, Genus, and } \\
\text { species }\end{array}$ & $\begin{array}{l}\text { Habitat } \\
\text { types }\end{array}$ & Threat to humans & $\begin{array}{l}\text { IUCN } \\
\text { status }\end{array}$ & $\begin{array}{l}\text { Trophic } \\
\text { level }\end{array}$ & $\begin{array}{l}\text { Other } \\
\text { remarks }\end{array}$ \\
\hline Xiphias gladius Linnaeus, 1758 & PEL; OD & & EN & 4.5 & $\mathrm{r} 1$ \\
\hline \multicolumn{6}{|l|}{ Family Istiophoridae } \\
\hline \multicolumn{6}{|l|}{ Genus Histiophofus } \\
\hline Istiophorus platypterus (Shaw, 1792) & PEL; OD & & LC & 4.5 & $\mathrm{r} 1$ \\
\hline \multicolumn{6}{|l|}{ Genus Istiompax } \\
\hline Istiompax indica (Cuvier, 1832) & PEL; OD & & & 4.5 & $\mathrm{r} 1$ \\
\hline \multicolumn{6}{|l|}{ Family Psettodidae } \\
\hline \multicolumn{6}{|l|}{ Genus Psettodes } \\
\hline $\begin{array}{l}\text { Psettodes erumei (Bloch \& Schneider, } \\
\text { 1801) }\end{array}$ & DEM & & & 4.4 & $\mathrm{r} 1$ \\
\hline \multicolumn{6}{|l|}{ Family Bothidae } \\
\hline \multicolumn{6}{|l|}{ Genus Bothus } \\
\hline Bothus mancus (Broussonet, 1782) & RFA & & LC & 4.4 & $\mathrm{r} 1$ \\
\hline Bothus pantherinus (Rüppell, 1830) & RFA & & LC & 3.5 & $\mathrm{r} 1$ \\
\hline \multicolumn{6}{|l|}{ Family Soleidae } \\
\hline \multicolumn{6}{|l|}{ Genus Aseraggodes } \\
\hline Aseraggodes dubius Weber, 1913 & DEM & & & 3.5 & r5 \\
\hline \multicolumn{6}{|l|}{ Order Tetraodontiformes } \\
\hline \multicolumn{6}{|l|}{ Family Balistidae } \\
\hline \multicolumn{6}{|l|}{ Genus Abalistes } \\
\hline Abalistes stellatus (Anonymous, 1798) & RFA & & & 3.4 & $r 1, r 7$ \\
\hline \multicolumn{6}{|l|}{ Genus Balistapus } \\
\hline Balistapus undulatus (Park, 1797) & & & & 3.4 & $\mathrm{r} 1$ \\
\hline \multicolumn{6}{|l|}{ Genus Balistes } \\
\hline Balistes rotundatus Marion de Procé, 1822 & RFA & Traumatogenic & & 3.5 & $\mathrm{r} 1$ \\
\hline \multicolumn{6}{|l|}{ Genus Balistoides } \\
\hline $\begin{array}{l}\text { Balistoides conspicillum (Bloch \& } \\
\text { Schneider, 1801) }\end{array}$ & RFA & $\begin{array}{l}\text { Reports of ciguatera } \\
\text { poisoning }\end{array}$ & & 3.3 & $\mathrm{r} 1$ \\
\hline $\begin{array}{l}\text { Balistoides viridescens (Bloch \& } \\
\text { Schneider, 1801) }\end{array}$ & RFA & $\begin{array}{l}\text { Reports of ciguatera } \\
\text { poisoning }\end{array}$ & & 3.3 & r2 \\
\hline Genus Melichthys & & & & & \\
\hline
\end{tabular}




\begin{tabular}{|c|c|c|c|c|c|}
\hline $\begin{array}{l}\text { Subclass, Order, Family, Genus, and } \\
\text { species }\end{array}$ & $\begin{array}{l}\text { Habitat } \\
\text { types }\end{array}$ & Threat to humans & $\begin{array}{l}\text { IUCN } \\
\text { status }\end{array}$ & $\begin{array}{l}\text { Trophic } \\
\text { level }\end{array}$ & $\begin{array}{l}\text { Other } \\
\text { remarks }\end{array}$ \\
\hline Melichthys niger (Bloch, 1786) & RFA & & LC & 2.4 & r2 \\
\hline Melichthys vidua (Richardson, 1845) & RFA & & & 3.4 & $r 1, r 11$ \\
\hline \multicolumn{6}{|l|}{ Genus Odonus } \\
\hline Odonus niger (Rüppell, 1836) & RFA & & & 3.2 & $\mathrm{r} 1$ \\
\hline \multicolumn{6}{|l|}{ Genus Pseudobalistes } \\
\hline $\begin{array}{l}\text { Pseudobalistes flavimarginatus (Rüppell, } \\
\text { 1829) }\end{array}$ & RFA & $\begin{array}{l}\text { Reports of ciguatera } \\
\text { poisoning }\end{array}$ & & 2.8 & $\mathrm{r} 1$ \\
\hline $\begin{array}{l}\text { Pseudobalistes fuscus (Bloch \& } \\
\text { Schneider, 1801) }\end{array}$ & RFA & & & 4.0 & $\mathrm{r} 1$ \\
\hline \multicolumn{6}{|l|}{ Genus Rhinecanthus } \\
\hline Rhinecanthus aculeatus (Linnaeus, 1758) & RFA & & & 3.2 & $\mathrm{r} 1, \mathrm{r} 11$ \\
\hline $\begin{array}{l}\text { Rhinecanthus rectangulus (Bloch \& } \\
\text { Schneider, 1801) }\end{array}$ & RFA & & & 3.5 & $\mathrm{r} 1, \mathrm{r} 11$ \\
\hline \multicolumn{6}{|l|}{ Genus Sufflamen } \\
\hline $\begin{array}{l}\text { Sufflamen chrysopterum (Bloch \& } \\
\text { Schneider, 1801) }\end{array}$ & RFA & & & 3.5 & $\mathrm{r} 1, \mathrm{r} 11$ \\
\hline Sufflamen fraenatum (Latreille, 1804) & RFA; OD & & LC & 3.7 & r3,r6 \\
\hline \multicolumn{6}{|l|}{ Genus Xanthichthys } \\
\hline $\begin{array}{l}\text { Xanthichthys lineopunctatus (Hollard, } \\
\text { 1854) }\end{array}$ & RFA & & & 3.5 & $\mathrm{r} 1$ \\
\hline $\begin{array}{l}\text { Xanthichthys auromarginatus (Bennett, } \\
1832 \text { ) }\end{array}$ & RFA & & & 3.0 & r9,r11 \\
\hline \multicolumn{6}{|l|}{ Family Monacanthidae } \\
\hline \multicolumn{6}{|l|}{ Genus Aluterus } \\
\hline Aluterus scriptus (Osbeck, 1765) & RFA & $\begin{array}{l}\text { Reports of ciguatera } \\
\text { poisoning }\end{array}$ & LC & 2.8 & $\mathrm{r} 1$ \\
\hline \multicolumn{6}{|l|}{ Genus Cantherhines } \\
\hline Cantherhines dumerilii (Hollard, 1854) & RFA & & LC & 3.1 & $r 1, r 6, r 11$ \\
\hline Cantherhines pardalis (Rüppell, 1837) & RFA & & LC & 3.5 & $\mathrm{r} 1, \mathrm{r} 11$ \\
\hline \multicolumn{6}{|l|}{ Genus Chaetodermis } \\
\hline Chaetodermis penicilligerus (Cuvier, 1816) & RFA & & LC & 2.8 & $\mathrm{r} 1$ \\
\hline Genus Oxymonacanthus & & & & & \\
\hline
\end{tabular}




\begin{tabular}{|c|c|c|c|c|c|}
\hline $\begin{array}{l}\text { Subclass, Order, Family, Genus, and } \\
\text { species }\end{array}$ & $\begin{array}{l}\text { Habitat } \\
\text { types }\end{array}$ & Threat to humans & $\begin{array}{l}\text { IUCN } \\
\text { status }\end{array}$ & $\begin{array}{l}\text { Trophic } \\
\text { level }\end{array}$ & $\begin{array}{l}\text { Other } \\
\text { remarks }\end{array}$ \\
\hline $\begin{array}{l}\text { Oxymonacanthus longirostris (Bloch \& } \\
\text { Schneider, 1801) }\end{array}$ & RFA & & VU & 3.3 & $\mathrm{r} 1, \mathrm{r} 6$ \\
\hline \multicolumn{6}{|l|}{ Genus Paraluteres } \\
\hline Paraluteres prionurus (Bleeker, 1851) & RFA & & LC & 2.7 & r6 \\
\hline \multicolumn{6}{|l|}{ Genus Pervagor } \\
\hline Pervagor janthinosoma (Bleeker, 1854) & RFA & & LC & 2.9 & $\mathrm{r} 1$ \\
\hline Pervagor melanocephalus (Bleeker, 1853) & RFA & & LC & 2.9 & $\mathrm{r} 1, \mathrm{r} 6$ \\
\hline \multicolumn{6}{|l|}{ Family Ostraciidae } \\
\hline \multicolumn{6}{|l|}{ Genus Lactoria } \\
\hline Lactoria cornuta (Linnaeus, 1758) & RFA & $\begin{array}{l}\text { Reports of ciguatera } \\
\text { poisoning }\end{array}$ & & 3.5 & $\mathrm{r} 1$ \\
\hline \multicolumn{6}{|l|}{ Genus Ostracion } \\
\hline Ostracion cubicus Linnaeus, 1758 & RFA & & & 3.4 & $\mathrm{r} 1, \mathrm{r} 6$ \\
\hline Ostracion meleagris Shaw, 1796 & RFA & Venomous & & 2.7 & r2 \\
\hline Ostracion rhinorhynchos Bleeker, 1851 & RFA & Venomous & & 3.5 & r2 \\
\hline \multicolumn{6}{|l|}{ Family Tetraodontidae } \\
\hline \multicolumn{6}{|l|}{ Genus Arothron } \\
\hline Arothron hispidus (Linnaeus, 1758) & RFA & Poisonous to eat & LC & 3.2 & $\mathrm{r} 1$ \\
\hline Arothron meleagris (Anonymous, 1798) & RFA & Poisonous to eat & LC & 3.6 & $\mathrm{r} 1$ \\
\hline $\begin{array}{l}\text { Arothron nigropunctatus (Bloch \& } \\
\text { Schneider, 1801) }\end{array}$ & RFA & Poisonous to eat & LC & 3.4 & $\mathrm{r} 1, \mathrm{r6}, \mathrm{r} 11$ \\
\hline Arothron stellatus (Anonymous, 1798) & RFA & Poisonous to eat & LC & 3.7 & r2 \\
\hline \multicolumn{6}{|l|}{ Genus Canthigaster } \\
\hline Canthigaster jactator (Jenkins, 1901) & RFA & & LC & 2.8 & $\mathrm{r} 1$ \\
\hline $\begin{array}{l}\text { Canthigaster rivulata (Temminck \& } \\
\text { Schlegel, 1850) }\end{array}$ & RFA & Venomous & LC & 3.1 & $\mathrm{r} 1$ \\
\hline Canthigaster valentini (Bleeker, 1853) & RFA & Poisonous to eat & LC & 2.8 & $\mathrm{r} 6, \mathrm{r} 11$ \\
\hline $\begin{array}{l}\text { Canthigaster coronata (Vaillant \& } \\
\text { Sauvage, 1875) }\end{array}$ & Rfa & Other & LC & 3.5 & $r 10, r 11$ \\
\hline Genus Lagocephalus & & & & & \\
\hline
\end{tabular}




\begin{tabular}{|c|c|c|c|c|c|}
\hline $\begin{array}{l}\text { Subclass, Order, Family, Genus, and } \\
\text { species }\end{array}$ & $\begin{array}{l}\text { Habitat } \\
\text { types }\end{array}$ & Threat to humans & $\begin{array}{l}\text { IUCN } \\
\text { status }\end{array}$ & $\begin{array}{l}\text { Trophic } \\
\text { level }\end{array}$ & $\begin{array}{l}\text { Other } \\
\text { remarks }\end{array}$ \\
\hline $\begin{array}{l}\text { Lagocephalus lagocephalus (Linnaeus, } \\
1758 \text { ) }\end{array}$ & BEP; OD & Other & LC & 3.7 & $\mathrm{r} 1$ \\
\hline Lagocephalus sceleratus (Gmelin, 1789) & RFA & Poisonous to eat & LC & 3.7 & $\mathrm{r} 1$ \\
\hline \multicolumn{6}{|l|}{ Genus Sphoeroides } \\
\hline $\begin{array}{l}\text { Sphoeroides pachygaster (Müller \& } \\
\text { Troschel, 1848) }\end{array}$ & BEP & & VU & 4.2 & r3 \\
\hline \multicolumn{6}{|l|}{ Family Diodontidae } \\
\hline \multicolumn{6}{|l|}{ Genus Chilomycterus } \\
\hline Chilomycterus reticulatus (Linnaeus, 1758) & RFA & Venomous & LC & 3.5 & r2 \\
\hline \multicolumn{6}{|l|}{ Genus Diodon } \\
\hline Diodon holocanthus Linnaeus, 1758 & RFA & $\begin{array}{l}\text { Reports of ciguatera } \\
\text { poisoning }\end{array}$ & LC & 3.9 & $\mathrm{r} 1$ \\
\hline Diodon hystrix Linnaeus, 1758 & RFA & Poisonous to eat & LC & 3.7 & $\mathrm{r} 1$ \\
\hline Diodon liturosus Shaw, 1804 & RFA & $\begin{array}{l}\text { Reports of ciguatera } \\
\text { poisoning }\end{array}$ & & 3.5 & r2 \\
\hline
\end{tabular}

Other abbreviations and references include: Institute, South China Sea Fisheries 1979 [r1], Li et al. 2007 [r2], Sun et al. 2004 [r3], Zeng 2004 [r4], Wang 1981 [r5], Gao et al. 2014 [r6], Wang et al. 2011 [r7], Yang et al. 2018 [r8], Huang 2018 [r9], Fu 2014 [r10] and the current study [r11].

\section{Taxon treatment}

\section{Dischistodus pseudochrysopoecilus Allen \& Robertson, 1974}

\section{Nomenclature}

Common name: Monarch damsel

\section{Material}

Holotype:

a. island: Xisha Island; country: China; stateProvince: Hainan

\section{Taxon discussion}

Maximum size of $18 \mathrm{~cm}$ Fig. 2. Dark brown (nearly black) with blue streak on each scale, blue lines and spots on head and white spot on middle of upper back. Coral thickets interspersed with open sand or dead coral in 1-5 m (Allen and Erdmann 2012). 


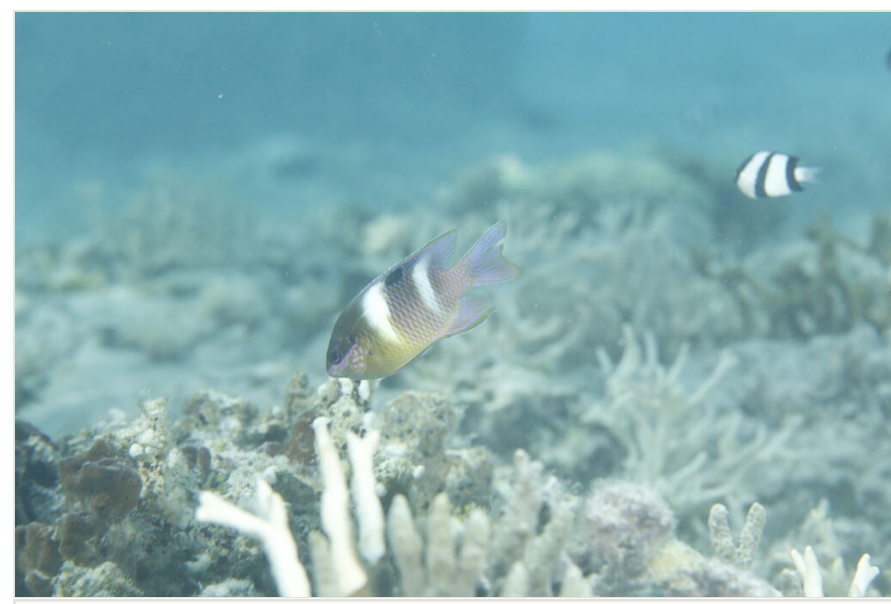

Figure 2. doi

Dischistodus pseudochrysopoecilus — a newly-recorded species in China

What we captured underwater in Xisha Islands is the state of the juvenile fish, which is very different from the adult fish. We have checked the main domestic fish checklists (Liu 2008, Chen and Zhang 2015, Shen and Wu 2011) and relevant literature, but there is no record of this species. Therefore, we believe it is a newly-recorded species in China.

\section{Discussion}

According to previous research, the order Perciforms is the most dominant order in the Xisha Islands (Li et al. 2007) and the major families in the Xisha Islands are Labridae and Pomacentridae (Gao et al. 2014) which concur with our study. Combined with studies over the years, in the past 20 years, Hyperoglyphe perciformis has been the dominant species in both underwater visual censuses and gillnet surveys.

The CFDI value for the Xisha Archipelago was 229, such that we could estimate put it into the formula proposed by Allen 1988, we estimated that Xisha Islands had 756 coral reef fishes. However, only 690 species were found, which is relatively comprehensive, but there is still a need for more surveys in different seasons within different sites in the future.

In term of the IUCN Red List Anonymous (2020a), one species is Critically Endangered ( Glyphis gangeticus); six species are Endangered (Stegostoma fasciatum, Aetomylaeus maculatus, Aetomylaeus vespertilio, Epinephelus akaara, Cheilinus undulatus and Xiphias gladius), 16 species are Vulnerable (Nebrius ferrugineus, Alopias vulpinus, Carcharhinus albimarginatus, Carcharhinus falciformis, Carcharhinus longimanus, Hemigaleus microstoma, Sphyrna lewini, Rhina ancylostoma, Rhynchobatus djiddensis, Taeniurops meyeni, Urogymnus asperrimus, Epinephelus fuscoguttatus, Plectropomus areolatus, Thunnus obesus, Oxymonacanthus longirostris and Sphoeroides pachygaster) and 13 species are Near Threatened in the Xisha Archipelago (Aetobatus narinari, Isurus 
oxyrinchus, Atelomycterus marmoratus, Carcharhinus amblyrhynchoides, Carcharhinus limbatus, Galeocerdo cuvier, Prionace glauca, Scoliodon laticaudus, Triaenodon obesus, Hexanchus griseus, Chaetodon trifascialis, Choerodon schoenleinii and Thunnus albacares). Thus, policy-makers and scientists should pay more attention to these species, by controlling coral reef degradation and overfishing and conducting coral reef restoration to strengthen the conservation of the fishes and the whole reef ecosystem. (Du et al. 2019). According to the data, $5.25 \%$ of the fish species are in the IUCN Red List, which is close to the number of the Redang Islands in Malaysia, at $5.1 \%$.

Reef associated fish (FRA) are the dominant type in the Xisha Islands, which have 500 species. They constitute $83 \%$ of the total fish. Other types of fishes make up less than $5 \%$ of the total fish in the Xisha Islands.

According to Pauly et al. (2002), a trophic level of more than 3.5 is considered a high-grade carnivorous fish and nearly half (306 species) of all fishes in the Xisha Islands belong to this type.

Intriguingly, we found some species (for example: Scolopsis aurata) that were recorded in the Xisha Islands (Zeng 2004), but according to the Computer-Generated Native Distribution Map from Fishbase, this species currently is distributed in the Indian Ocean: Maldives, Sri Lanka and southern Indonesia. This may support the hypothesis of species shifting northwards, but more research is needed.

\section{Acknowledgements}

This study was supported by National Key Research and Development Program of China (No. 2018YFC1406503), the National Natural Science Foundation of China (No. 41676096), the China-ASEAN Maritime Cooperation Fund project "Monitoring and conservation of the coastal ecosystem in the South China Sea" and Top 100 Universities in the World Fund (TU001-2018).

\section{Author contributions}

Design research: ST Qiu, B Chen, JG Du, and KH Loh

Acquisition of funding: JG Du, B Chen

Analysis of data: ST Qiu and JG Du

Writing (original manuscript): ST Qiu and JG Du

Writing (review and editing): J Du, B Chen, KH Loh, XM liu, W Yang 


\section{References}

- $\quad$ Allen GR (1988) Reef and shore fishes of Milne Bay Province, Papua New Guinea. In: Werner TB, Allen GR (Eds) A rapid biodiversity assessment of the coral reefs of Milne Bay Province, Papua New Guinea.

- $\quad$ Allen GR (2008) Conservation hotspots of biodiversity and endemism for Indo-Pacific coral reef fishes. Aquatic Conservation: Marine and Freshwater Ecosystems 18: 541-556.

- $\quad$ Allen GR, Erdmann MV (2012) Reef fishes of the East Indies. Volumes I-III. Tropical Reef Research, Perth, Australia. University of Hawaii Press

- $\quad$ Anonymous (2020) The IUCN Red List of Threatened Species. https://www.iucnredlist.org.

- $\quad$ Chen DG, Zhang MZ (2015) Marine Fishes of China I III. China Ocean University Press [ISBN 978-7-5670-1065-9]

- $\quad$ Du JG, Luo K, Hu WJ, Zheng xq, et al. (2019) An updated checklist of the marine fish fauna of Redang Islands, Malaysia. Biodiversity Data Journal 7: e47537. https://doi.org/ 10.3897/BDJ.7.e47537

- $\quad$ Fang HD, Lv XL (2019) Reef fish identification of Nansha Islands. China Ocean University Press

- $\quad$ Froese R, Pauly D (2020) www.fishbase.de

- $\quad$ Fu L (2014) Coral reef fishes of the South China Sea. China CITIC Press

- Gao YL, Huang H, Lian JS, Yang JH (2014) Species diversity and trophic structure of reef fishes in the waters of the Xisha Archipelago. Biodiversity Science 22 (5): 618-623. https://doi.org/10.3724/SP.J.1003.2014.14102

- Huang H (2018) Coral reef atlas of Xisha Islands. Science Press https://doi.org/ 10.1007/s00338-018-1724-8

- Huang L, Huang H, Jiang L (2020) A revised taxonomy for Chinese hermatypic corals. Biodiversity Science 28 (4): 515-523. https://doi.org/10.17520/biods.2019384

- Institute, South China Sea Fisheries (1979) The fishes of the Islands in the South China Sea. Science Press, Beijing.

- $\quad$ Liu RY (2008) Checklist of Marine Biota of China Seas. Science Press [ISBN 978-7-03-023722-4]

- $\quad$ Li YC, Wu ZJ, Chen SQ, et al. (2017) Discussion of the diversity of the coral reef fish in the shallow reefs along the Yongxing and Qilianyu Islands. Marine Environmental Science (China) 36 (4): 509-516.

- $\quad$ Li YZ, Jia Xp, Chen GB, et al. (2007) The fishery resources on the reefs of South China Sea. China Ocean Press

- Pauly D, Christensen V, Gunette S, Pitcher TJ, Sumaila UR, Walters CJ, Watson R, Zeller D (2002) Towards sustainability in world fisheries. Nature 418: 689-695. https://doi.org/10.1038/nature01017

- Shen SJ, Wu GY (2011) Fishes of Taiwan. National Museum of Marine Biology \& Aquarium [ISBN 978-986-02-9899-4]

- $\quad$ Sun DR, Lin SJ, Qiu YS, et al. (2004) Fish fauna of coral reef waters of the Xisha Islands. South China Fisheries Science

- Wang CX (1981) Studies on the fish fauna of the South China Sea islands, Guangdong Province, China. Science Press, Beijing 
- Wang XH, Du YF, Lin SJ, Sui D, Qiu YS, Huang SL (2011) Fish species diversity and community pattern in coral reefs of the Xisha Islands, South China Sea. Biodiversity Science 19 (4): 463-469. https://doi.org/10.3724/SP.J.1003.2011.07267

- Yang WD, Hu JT, Lin BA, Huang H, Liu M (2018) Diversity of coral reef fishes in Zhaoshu Island waters, Xisha Islands. Journal of Xiamen University (Natural Science) 57 (6): 819-826.

- Zeng XG (2004) The research on biological resources in the Waters of Main Islands and reefs of South China Sea. China Ocean Press 